\title{
Resolving few-layer antimonene/graphene heterostructures
}

\author{
Tushar Gupta $\mathbb{D}^{1}$, Kenan Elibol $\mathbb{\mathbb { D }}^{2}$, Stefan Hummel ${ }^{2,3}$, Michael Stöger-Pollach ${ }^{4}$, Clemens Mangler ${ }^{2}$, Gerlinde Habler $\mathbb{1}^{5}$, \\ Jannik C. Meyer $\mathbb{D}^{2}$, Dominik Eder $\left(\mathbb{D}^{1 凶}\right.$ and Bernhard C. Bayer $\mathbb{D}^{1,2 \bowtie}$
}

Two-dimensional (2D) antimony ( $\mathrm{Sb}$, "antimonene") is of interest in electronics and batteries. Sb however exhibits a large allotropic structural diversity, which is also influenced by its support. Thus, Sb heterostructure formation is key in 2D Sb integration. Particularly, 2D Sb/graphene interfaces are important. We thus study here few-layered 2D Sb/graphene heterostructures with atomic resolution (scanning) transmission electron microscopy. We find two $\mathrm{Sb}$ morphologies to coexist: first, a 2D morphology of layered $\beta$-Sb with $\beta$-Sb(001) ||graphene(001) texture. Second, one-dimensional Sb nanowires which can be matched to $\beta$-Sb[221] $\perp$ graphene(001) and are closely related to cubic $\mathrm{Sb}(001) \|$ graphene(001). Importantly, both $\mathrm{Sb}$ morphologies show rotational van-der-Waals epitaxy with graphene. Both are resilient against oxidation, although superficial Sb-oxide formation merits consideration, including epitaxial $\mathrm{Sb}_{2} \mathrm{O}_{3}(111) / \beta-\mathrm{Sb}(001)$ heterostructures. Exact $\mathrm{Sb}$ growth behavior depends on processing and substrate properties including, notably, the support underneath the graphene. Our work elucidates the rich phase and epitaxy landscape in $2 \mathrm{D} \mathrm{Sb}$ and $2 \mathrm{D} \mathrm{Sb} /$ graphene heterostructures.

npj 2D Materials and Applications (2021)5:53; https://doi.org/10.1038/s41699-021-00230-3

\section{INTRODUCTION}

Among the two-dimensional (2D) pnictogens (i.e., group 15/VA elements, including $\mathrm{P}, \mathrm{As}, \mathrm{Sb}$, and $\mathrm{Bi})^{1-4}$ particularly mono- and few-layered 2D Sb ("antimonene") has recently attracted increasing research interest ${ }^{5,6}$. Firstly, this is due to $2 \mathrm{D}$ Sb's peculiar electronic properties toward novel 2D electronics including layer-dependent (semi-)metal-to-semiconductor transition ${ }^{7,8}$ high carrier mobilities ${ }^{9}$, strain-tunable indirect-to-direct band gap transition ${ }^{7,8}$, and the possibility of 2D and 3D topological insulator behavior for mono- ${ }^{10}$ and few-layered ${ }^{5} 2 \mathrm{D} \mathrm{Sb}$, respectively. Secondly, the recent high interest equally results from $2 \mathrm{D}$ Sb's high suitability for sustainable energy and catalysis applications, including as an anode material in next-generation $\mathrm{Li}$ - and $\mathrm{Na}$-ion batteries ${ }^{11-21}$ as well as for (electro) catalysis ${ }^{22-24}$, supercapacitors ${ }^{25}$, charge extraction in photovoltaics $^{26}$, and thermoelectrics ${ }^{27}$.

$\mathrm{Sb}$ however shows a large allotropic/polymorphic structural diversity. This includes several layered, potential 2D forms $s^{8,28-31}$ such as in particular the thermodynamically most stable, rhombohedral, buckled honeycomb-structured $\beta$-Sb (A7, R-3m, $166)^{22,23,30-44}$ and the metastable orthorhombic, puckered "wash-

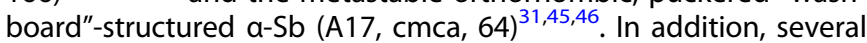
nonlayered metastable allotropes at high pressure and in thin film form have been reported including simple cubic, body-centeredcubic, face-centered-cubic, and hexagonally close-packed $\mathrm{Sb}^{47-56}$. Most of these phases are related via small atomic rearrangements ${ }^{31,54-56}$, and some even have been suggested to show thickness-dependent phase transitions in nanostructures ${ }^{31}$. This polymorphicity calls for close control over Sb's structure in any potential synthesis scenario for the various desired application fields. The structure of $\mathrm{Sb}$ deposits is however not only determined by kinetic growth process conditions but is also intimately linked to the Sb deposits' support, an effect which is exacerbated for ultrathin 2D Sb. Therefore, understanding $\mathrm{Sb}$ heterostructure formation is key for controllable 2D Sb growth. This is true not only for Sb's use in 2D electronics, where typically laterally large, defect-free 2D Sb films are desired ${ }^{33}$, but also for $\mathrm{Sb}^{\prime} \mathrm{s}$ use in catalysis and energy applications, where often nanosized 2D Sb deposits with a large number of edges are preferred $^{22,23}$.

Among the various possible $\mathrm{Sb}$ heterostructures ${ }^{5}$, in particular, 2D Sb/graphene interfaces are of prime importance for two reasons: first, in the context of catalysis and energy applications, Sb/carbon hybrids are emerging as a highly useful materials combination, e.g. in batteries ${ }^{11-20}$, electrocatalysis ${ }^{22,23}$, and supercapacitors ${ }^{25} .2 \mathrm{D} \mathrm{Sb} /$ graphene heterostructures can readily approximate such $\mathrm{Sb} / \mathrm{carbon}$ hybrids in order to understand their as-of-yet little elucidated interface properties. Second, in the context of 2D electronics, recent work has suggested that device contacts formed by 2D Sb/ graphene heterostructures could be technologically advantageous toward tuning contact resistances ${ }^{57,58}$.

The structural properties and formation mechanisms of $\mathrm{Sb}$ heterostructures including $2 \mathrm{D} \mathrm{Sb/graphene,} \mathrm{remain} \mathrm{however} \mathrm{as-}$ of-yet largely underexplored, in particular at the atomically resolved level. To address this, we provide here an atomic-scale (scanning) transmission electron microscopy ((S)TEM) investigation into the properties of a few-layered 2D Sb/graphene heterostructure model system that, as we find, readily emulates $\mathrm{Sb} /$ carbon heterostructures as manufactured by vapor phase techniques for electronics ${ }^{38,48,50,51}$ and also as synthesized by wetchemistry routes for energy applications ${ }^{12,14,15,19,20}$. Our approach thereby facilitates direct assessment of interfacing and epitaxial effects in 2D Sb/carbon heterostructures, with graphene also acting as an ideal support ${ }^{59}$ for the employed atomic resolution (S) TEM techniques ${ }^{60}$.

Our work reveals in our 2D Sb/graphene heterostructures the coexistence of a $2 \mathrm{D}$ growth morphology of layered $\beta-\mathrm{Sb}(001) \|$ graphene(001) phase and texture as well as of a one-dimensional (1D) Sb growth morphology. The latter 1D morphology can be matched not only to $\beta$-Sb with $\beta$-Sb[2-21] $\perp$ graphene(001) texture but also to a nonlayered, thermodynamically nonpreferred cubic

\footnotetext{
${ }^{1}$ Institute of Materials Chemistry, Vienna University of Technology (TU Wien), Vienna, Austria. ${ }^{2}$ Faculty of Physics, University of Vienna, Vienna, Austria. ${ }^{3} \mathrm{GETec}$ Microscopy GmbH, Vienna, Austria. ${ }^{4}$ USTEM, Vienna University of Technology (TU Wien), Vienna, Austria. ${ }^{5}$ Department of Lithospheric Research, University of Vienna, Vienna, Austria.

${ }^{凶}$ email: dominik.eder@tuwien.ac.at; bernhard.bayer-skoff@tuwien.ac.at
} 
$\mathrm{Sb}(001)$ ||graphene(001). Importantly, both Sb morphologies show preferred relative crystallographic (mis)orientations with respect to the supporting graphene monolayer lattice, indicating that rotational van-der-Waals (vdW) epitaxy can readily exist in 2D Sb/ graphene heterostructures. Both Sb morphologies are found to be well resilient against environmental oxidation in ambient atmosphere although superficial surface oxidation is shown to be important to consider, particularly due to here suggested formation of epitaxial $\mathrm{Sb}_{2} \mathrm{O}_{3}(111) / \beta-\mathrm{Sb}(001)$ heterostructures as top layer. We find that exact $\mathrm{Sb}$ growth results are sensitive on employed processing techniques and substrate properties including, notably, the nature of the support underneath the direct graphene support. Our work thereby provides fundamental insights into the rich phase and epitaxy relations in $2 \mathrm{D} \mathrm{Sb}$ and 2D Sb/graphene heterostructures.

\section{RESULTS AND DISCUSSION}

\section{Morphology and structure}

We first characterize in Fig. 1 the morphology and structure of the few-layer Sb on graphene model system, which is prepared by physical vapor deposition (PVD) of $\mathrm{Sb}$ onto chemical vapor deposited (CVD) monolayer graphene. We first focus on optimized $\mathrm{Sb}$ deposition conditions toward high Sb crystallinity, with the wider parameter space of the Sb PVD on graphene being discussed further below. The nominal $10 \mathrm{~nm}$ thick Sb deposits in Figs. 1-4 were thermally evaporated onto monolayered CVD graphene films ${ }^{61,62}$. During Sb PVD (base pressure $\sim 10^{-5} \mathrm{mbar}$ ), the graphene substrates were held at room temperature (RT) and also at controlled temperatures of 150 and $250^{\circ} \mathrm{C}$. The graphene either remained on its Cu CVD catalyst foils ${ }^{61,62}$ during Sb PVD (Figs. 1a, b and 2) or was additionally also transferred prior to $\mathrm{Sb}$ PVD to be suspended as a freestanding monolayer membrane across holey TEM grids ${ }^{63}$ (i.e., no Cu foils underneath, Figs. 1c-g, 3, and 4). Nominal deposited Sb thickness was measured via a coexposed (nonheated) quartz crystal microbalance (QMB). After $\mathrm{Sb}$ deposition, samples were stored in ambient air.

The scanning electron microscopy (SEM) image of the $250^{\circ} \mathrm{C}$ deposition in Fig. 1a reveals that under our optimized PVD conditions, the Sb deposits on the graphene form isolated islands with two distinctly different base shapes: first are flat 2D Sb deposits with (truncated) hexagonal or (truncated) triangular base shapes. Second are rod-like 1D Sb deposits with rectangular bases. Lateral extents of all Sb deposits are in the range of tens to hundreds of $\mathrm{nm}$. While such lateral sizes are small for device fabrication in 2D electronics, they are well compatible with the requirements for $2 \mathrm{D} \mathrm{Sb}$ catalysis and energy applications ${ }^{22}$. Importantly, such feature sizes also provide a convenient $\mathrm{Sb} /$ graphene heterostructure model system for elucidation of $\mathrm{Sb}$ phases and interfacing by high-resolution (S)TEM. Notably, as shown in Fig. 1a, the edges of both the triangular-/hexagonalshaped and the rod-shaped Sb deposits show a high degree of visually apparent directional alignment amongst each phase type, respectively. This is a first indication of potential epitaxy effects between the Sb deposits and their graphene support and will be further examined below.

The Raman spectrum corresponding to the $250^{\circ} \mathrm{C}$ deposition in Fig. $1 \mathrm{~b}$ displays primarily two peaks at low wavenumbers that are characteristic for elemental Sb $\left(117 \mathrm{~cm}^{-1} ; 154 \mathrm{~cm}^{-1}\right)$. These peaks are best matched with $E_{g}$ and $A_{1 g}$ modes of few-layer $\beta$-Sb, respectively, but are also potentially consistent with $\mathrm{a}$-Sb and/or pressure-induced phases of $\mathrm{Sb}^{8,31,33,46,54}$. We note that thicker $\mathrm{Sb}$ deposits may be overrepresented in Raman intensity ${ }^{42,43}$. Significant volume Sb-oxide formation can be excluded based on our Raman data as the signal intensity at wavenumbers corresponding to $\mathrm{Sb}$ oxides is comparatively weak (e.g., for thermodynamically most stable $\mathrm{Sb}_{2} \mathrm{O}_{3}$ expected at $\sim 190$ and $\left.250 \mathrm{~cm}^{-1}\right)^{64}$. The minor peak at $\sim 215 \mathrm{~cm}^{-1}$ is related to minor $\mathrm{Cu}$ oxide formation on bare regions of the $\mathrm{Cu}$ support during ambient air storage ${ }^{65,66}$. Raman peaks characteristic for graphene $(G$ at $1593 \mathrm{~cm}^{-1}$ and $2 \mathrm{D}$ at $2701 \mathrm{~cm}^{-1}$ ) are also found in Fig. $1 \mathrm{~b}$, consistent with the high quality CVD graphene used as substrate $^{61,62}$. The absence of a significant defect-related $D$ peak at $\sim 1350 \mathrm{~cm}^{-1}$ confirms that the CVD graphene support was not degraded during Sb PVD. Thereby our Raman data also confirm that no covalent $\mathrm{Sb}$-carbon bond formation has occurred and that our 2D Sb/graphene interfaces are of vdW type ${ }^{38}$, consistent with theoretical predictions ${ }^{8,57,58}$.

To assess the crystallographic structure of the Sb deposits in a localized fashion, we employ in Fig. 1c-g aberration-corrected, atomically resolved and element-specific STEM (Nion UltraSTEM 100 at $60 \mathrm{kV}$ electron acceleration voltage) in annular dark field $(A D F)^{60}$ mode to image individual Sb deposits at high resolution in top plan view. Corresponding ADF STEM and bright-field (BF) TEM data from focused ion beam (FIB) cross sections in Fig. 2 provide a complementary side view of the Sb deposits. Supplementary Figs. 2 and 3 provide atomic models and Fourier transform (FT)/ selected area electron diffraction (SAED) simulations of all identified phases. We note that under our STEM imaging conditions no electron beam induced phase transitions or materials modifications to the Sb deposits were observed ${ }^{67-69}$.

The first group of interest is the flat $\mathrm{Sb}$ deposits from Fig. 1a with (truncated) hexagonal (Fig. 1c) or triangular base shape (Fig. 1d). The phase identification for these structures is straightforward: at atomic resolution and view from top, all flat hexagonal/triangular deposits show a sixfold symmetric appearance that can be best indexed via the FT of their atomic resolution images to rhombohedral $\beta$-Sb viewed along the [001] zone axis (Fig. 1h), i.e., with the basal (001) layers of the layered 2D $\beta$-Sb parallel to the graphene(001) substrate (i.e., $\beta$-Sb[001] $\perp$ graphene $(001)=\beta-S b(001) \|$ graphene(001)). This phase identification to $\beta-\mathrm{Sb}(001)$ is also fully corroborated by the corresponding side view of a triangular/hexagonal deposits in Fig. $2 b-d$, which clearly resolves the layered nature of the $\beta-\mathrm{Sb}(001)$ when viewed along the [110] zone axis, with the $\beta-\mathrm{Sb}(001)$ planes parallel to the graphene(001) substrate. Delineating projected edge directions correspond to [100], [010], and [110] in the top view STEM images for both hexagonal and triangular $\beta$-Sb (Fig. 1c, d). Hexagonal and triangular deposits typically appear flat in STEM images, indicating (001) top surfaces. The observation of $2 \mathrm{D} \beta-\mathrm{Sb}(001) \|$ graphene $(001)$ is in line with recent literature ${ }^{31-39}$.

The second group of interest is the rod-like 1D Sb deposits with rectangular bases (Fig. 1e-g). In top view at atomic resolution, these structures always show a FT with fourfold symmetry. Their phase identification is less straightforward: on the one hand, the STEM data agree with $\beta$-Sb when viewed along the [2-21] zone axis, i.e., at a texture of $\beta-S b[2-21] \perp$ graphene(001). Notably, $\beta-S b$ with [2-21] zone axis perpendicular to support does not have a defined low (hkl) value interface plane parallel to the support when viewed from the side, but only slightly inclined base planes (Fig. 1i; an approximation for an interface plane would be $\beta-S b\left(\begin{array}{lll}10 & -10 & 23) \text { ). }\end{array}\right.$ $\beta-S b[2-21]$ is closely related to AA-stacked $a-S b$ multilayers via a small shear deformation ${ }^{31}$. Recently, a thickness-dependent crossover from $a-S b$ to $\beta-S b[2-21]$ has been suggested to occur in 1D Sb deposits $^{31}$. On the other hand, the fourfold symmetry STEM images of the $1 \mathrm{D}$ rod-like $\mathrm{Sb}$ deposits also matches well with a thermodynamically nonpreferred simple cubic, nonlayered Sb polymorph viewed along its [001] zone axis (Fig. 1j), i.e., cubic Sb $[001] \perp$ graphene $(001)=$ cubic $\mathrm{Sb}(001) \|$ graphene $(001)^{47,50}$. Cubic $\mathrm{Sb}$ polymorphs are related to rhombohedral $\beta$-Sb via a unidirectional deformation ${ }^{54-56}$. While the existence of cubic Sb in bulk form has been a long-standing matter of debate in the literature ${ }^{52-56}$, cubic Sb is typically associated with high pressure conditions but has also been reported to occur in Sb thin films, presumably formed via substrate-induced stress ${ }^{47-56}$. Notably, structurally $\beta$-Sb 
(a)

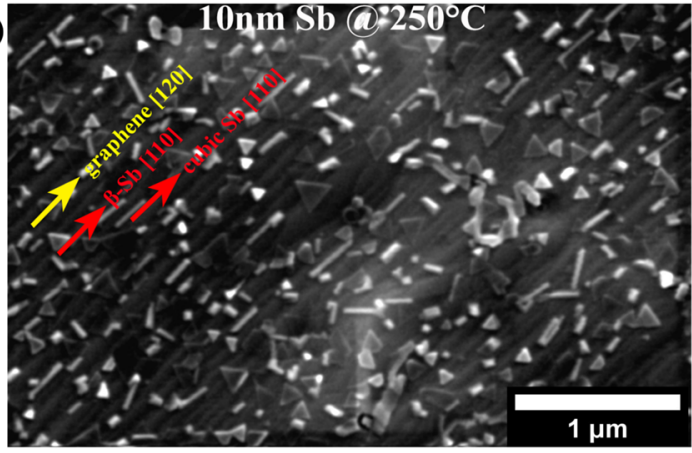

(c)
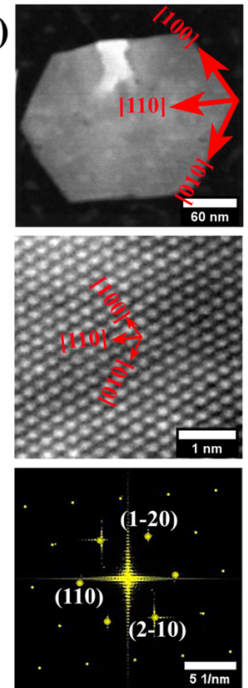

(h) $\beta$-Sb (001)

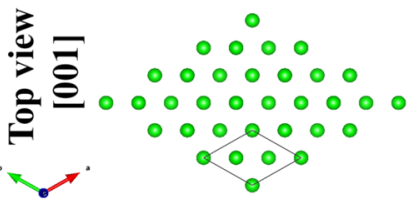

党

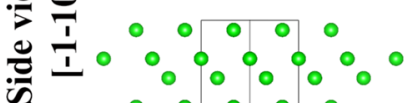

$\stackrel{0}{\infty} 0_{0}^{0} 0_{0}^{0} 0_{0}^{\circ}$

(e)

(i) (b)
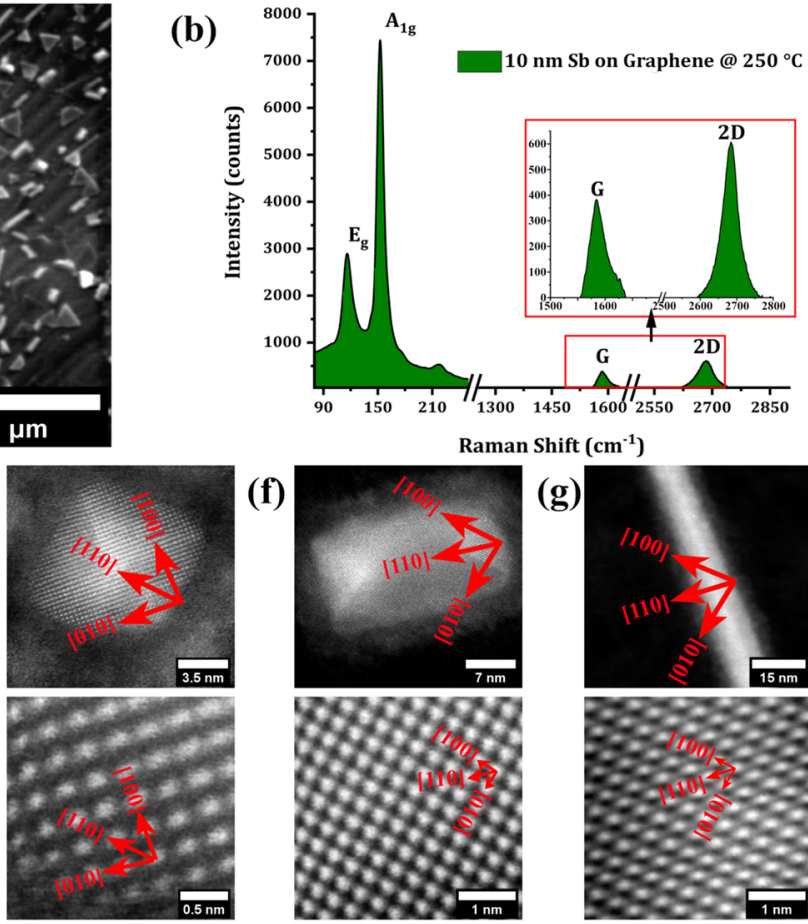

(f)

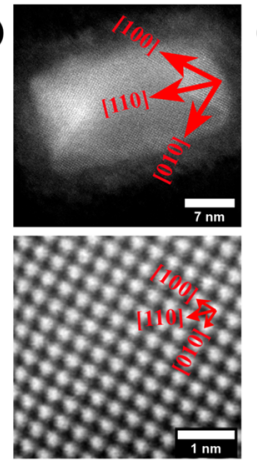

(g)
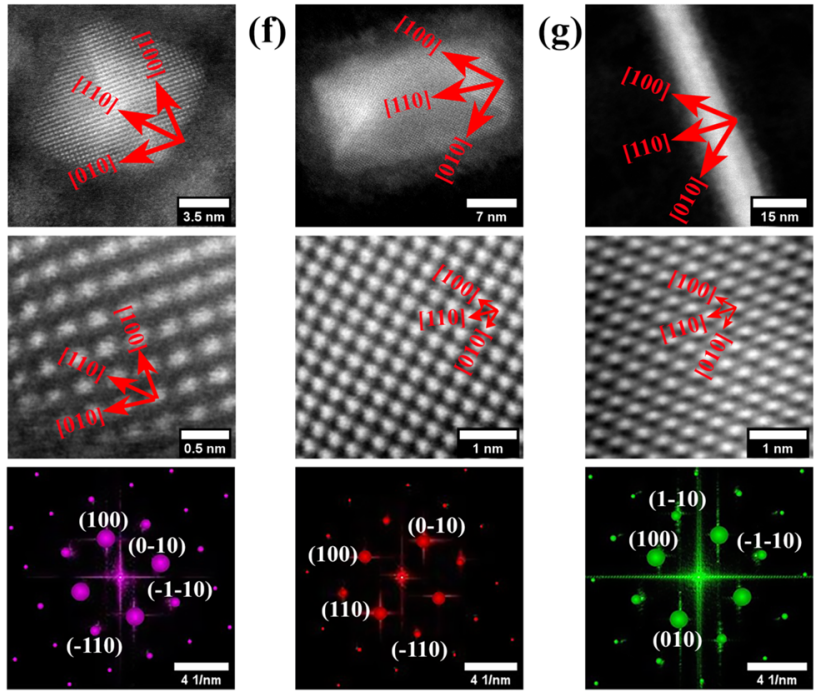

(j)

cubic Sb (001)
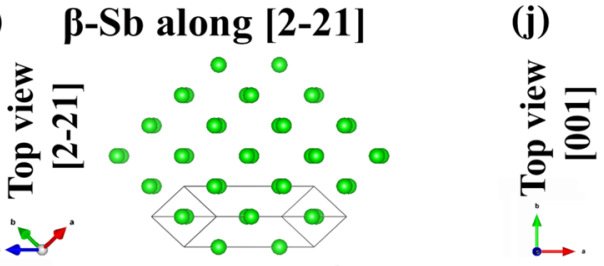

- $\circ \circ \circ 0$

- 0 ○ 0

- $000 \%$

- 0 ○ 0

0.000

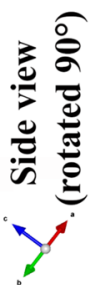

- 0000

- 0000

- 0000

0000
- $\odot \circ \circ \circ$

Fig. 1 Morphology and structure. a SEM image and b Raman spectrum of $10 \mathrm{~nm}$ Sb deposited at $250^{\circ} \mathrm{C}$ onto Cu-supported CVD graphene. Salient lattice directions in the graphene and the $\mathrm{Sb}$ are labeled in $\mathbf{a}$, as identified in the main text. c-g ADF STEM images of individual particles of $10 \mathrm{~nm} \mathrm{Sb}$ deposited at 150 and $250^{\circ} \mathrm{C}$ onto suspended monolayer graphene, showing overview (top) and atomic resolution images (middle) and corresponding FTs (bottom). The FTs are indexed to $\beta$-Sb(001) viewed along [001] zone axis (c, d) and cubic $\mathrm{Sb}(001)$ viewed along [001] zone axis (e-g), respectively. Corresponding salient crystallographic directions are superimposed over the ADF images. The sixfold symmetric "**-indexed reflection set in $\mathbf{d}$ is ascribed cubic $\mathrm{Sb}_{2} \mathrm{O}_{3}$ viewed along the [111] zone axis and corresponds to $\mathrm{Sb}_{2} \mathrm{O}_{3}(2-20)$ reflection family, as described below. False-color coded ADF STEM images of overview and atomic resolution images can be found in Supplementary Fig. 1. h-j Atomic models of $\beta$ - $\mathrm{Sb}(001), \beta-\mathrm{Sb}[2-21]$, and cubic $\mathrm{Sb}(001)$, respectively. For further information on atomic models and FT simulations see Supplementary Figs. 2 and 3.

[2-21] (and AA a-Sb multilayers) and cubic $\mathrm{Sb}(001)$ structures are all closely related and may gradually transition into each other ${ }^{31,54-56}$. This makes their differentiation difficult and partly ambiguous. This is also underlined by, e.g., the cross-sectional TEM of a rod-like Sb deposit in Fig. $2 \mathrm{~d}$ which shows lattice planes with a principal spacing of $\sim 0.3 \mathrm{~nm}$. These are consistent with $\beta-S b[2-21]$ as well as cubic $\mathrm{Sb}(001)$ viewed from the side (Fig. $1 \mathrm{i}, \mathrm{j}$ ). Therefore, for the remainder of this report, we refer to the 1D Sb morphology as " $\beta-\mathrm{Sb}[2-21] /$ cubic $\mathrm{Sb}(001)$ " phase and texture, suggesting that the $1 \mathrm{D}$ rods are compatible with both $\beta-\mathrm{Sb}[2-21] \perp$ graphene $(001)$ and cubic $\mathrm{Sb}(001) \|$ graphene(001). For determination of $\beta-S b[2-$ $21] /$ cubic $\mathrm{Sb}(001)$ in-plane epitaxial relations to graphene support, delineating projected edge directions and similar discussion below we will predominantly use the cubic Sb unit cell and associated (hkl) plane and [uvw] direction notation since this conveniently simplifies the description of the crystallographic 

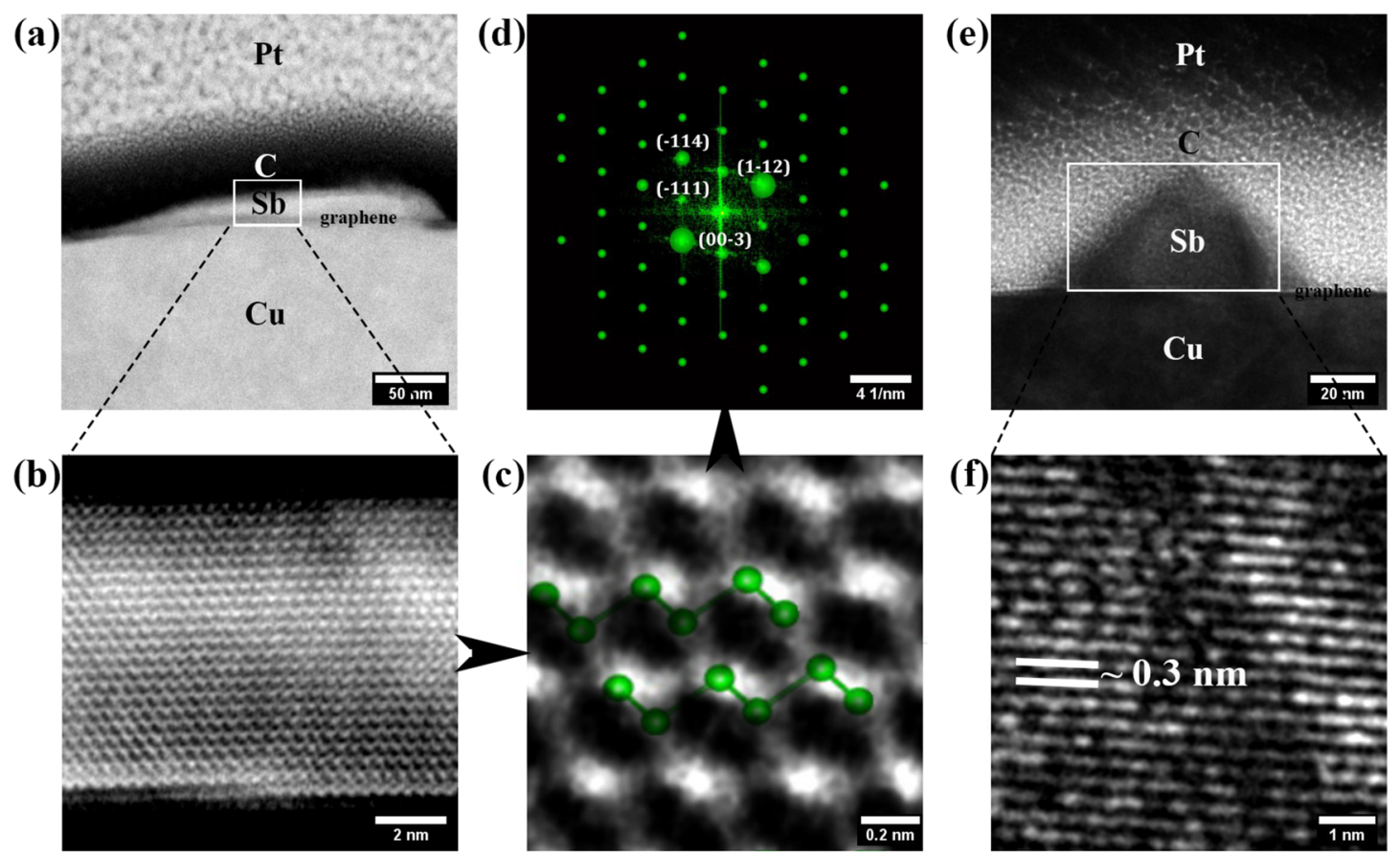

Fig. 2 Cross-sectional STEM/TEM of $10 \mathbf{~ n m ~ S b ~ d e p o s i t e d ~ o n t o ~ C u - s u p p o r t e d ~ g r a p h e n e . ~ A D F ~ S T E M ~ o f ~ a ~} \beta$-Sb(001) deposit in overview (a), intermediate (b), and high resolution (c). d The FT of $\mathbf{c}$ indexed to $\beta$-Sb(001) viewed along the [110] zone axis. In $\mathbf{c}$, a schematic of the $\beta$-Sb(001) layers is superimposed as illustration. BF-TEM of a $\beta-\mathrm{Sb}[2-21] / c u b i c ~ S b(001)$ deposit in overview (e) and at high resolution (f).

system compared to the inclined plane $\beta-S b[2-21]$ description. Delineating projected edge directions in the top view STEM images are cubic Sb [110] for the rods (Fig. 1e-g). Consequently, the rods long axis is cubic Sb [110] direction. Figures $1 \mathrm{e}-\mathrm{g}$ and $2 \mathrm{e}$ show that the 1D morphology does not have a flat top surface but rather very strong faceting to a pyramidal shape over a square (Fig. 1e) or rectangular (Fig. 1f, g) base. To best reproduce the observed angles in Figs. $1 \mathrm{e}-\mathrm{g}$ and $2 \mathrm{e}$, the delineating faceted surfaces have to be of (223) family in cubic $\mathrm{Sb}$ notation (Supplementary Fig. 4). Alternatively, also (111) family facets can provide a reasonable match. 1D Sb morphologies with fourfold atomic symmetries that coexist with 2D $\beta$ - $\mathrm{Sb}(001)$ have been observed in older work on Sb/graphite ${ }^{50,51}$ and also recent work on $\mathrm{Sb} /$ graphene ${ }^{31}$, albeit other recent work under very similar conditions for $\mathrm{Sb} /$ graphene heterostructures did not observe 1D nanostructure growth ${ }^{38}$. We note that one recent paper ${ }^{70}$ ascribed 1D Sb nanostructures to $\beta-\mathrm{Sb}(001)$, albeit without providing direct crystalline structure confirmation for their assignment.

We have confirmed the morphology-structure relation of triangular/hexagonal base shape corresponding to layered 2D $\beta-\mathrm{Sb}(001)$ and rectangular rod base shape corresponding to $\beta-\mathrm{Sb}$ [2-21]/cubic $\mathrm{Sb}(001)$ via $>60$ atomic resolution STEM, lattice resolution TEM, and SAED observations. This makes us confident that we can safely assign the crystallographic phase of a deposit via its macroscopic base shape as observed in lower magnification SEM or atomic force microscopy (AFM) data ${ }^{51}$.

Following this approach, AFM data (taken via conventional AFM as well as via correlated AFM-SEM, GETec AFSEM ${ }^{71}$, see Supplementary Fig. 5) indicate for depositions at $250^{\circ} \mathrm{C}$, for the layered $2 \mathrm{D} \beta-\mathrm{Sb}(001)$ deposits, a minimum thickness of $4.7 \mathrm{~nm}$ (equivalent to $\sim 12$ layers $s^{8,29,42}$, i.e., few-layer antimonene), and an average thickness ( \pm standard deviation) of $21 \pm 14 \mathrm{~nm}$. The 1D rod-like $\beta$-Sb[2-21]/cubic $\mathrm{Sb}(001)$ deposits are relatively thicker, with a minimum thickness of $10 \mathrm{~nm}$ and an average thickness of $31 \pm 10 \mathrm{~nm}$. To estimate the relative abundance of $2 \mathrm{D} \beta-\mathrm{Sb}(001)$ and 1D $\beta-\mathrm{Sb}[2-21] /$ cubic $\mathrm{Sb}(001)$ in our deposits, we compute from SEM and AFM images for the $250^{\circ} \mathrm{C}$ depositions, both domain number counts and average equivalent feature sizes (see "Methods" section for calculation) for each phase: via this analysis, we find a lower number fraction of $2 D \beta-S b(001)$ domains (40 \pm 1 count-\%) compared to $\beta$-Sb[2-21]/cubic $\mathrm{Sb}(001)(60 \pm 1$ count-\%). These $2 \mathrm{D} \beta-\mathrm{Sb}(001)$ domains grow to however cover a relatively larger area $(60 \pm 5$ area- $\%$ for $2 D \beta-S b(001)$ vs. $40 \pm 5$ area-\% for $\beta-\mathrm{Sb}[2-21] /$ cubic $\mathrm{Sb}(001))$. The observation that the $2 \mathrm{D}$ $\beta-\mathrm{Sb}(001)$ islands grow to larger lateral sizes is also reflected in a larger maximum and average equivalent feature size for the 2D $\beta-\mathrm{Sb}(001)$ (maximum: $260 \mathrm{~nm}$; average: $113 \pm 95 \mathrm{~nm}$ ) compared to the $\beta-S b[2-21] / c u b i c ~ S b(001)$ (maximum: $130 \mathrm{~nm}$; average: $72 \pm$ $54 \mathrm{~nm})$.

Comparing our results with the prior literature, we note that overall morphology and size of our $\mathrm{Sb}$ domains on carbon substrates are consistent not only with vacuum-based vapor deposition techniques as usually used in electronics ${ }^{38,48,50,51}$ (akin to our PVD synthesis) but also with several wet-chemistry synthesis routes (including using $\mathrm{SbCl}_{3}{ }^{12,14,19,20}$ and ball-milled and annealed $\mathrm{Sb} /$ carbon mixtures $^{15}$ ) as usually used in energy materials synthesis. This highlights that our here investigated $2 \mathrm{D}$ $\mathrm{Sb} / \mathrm{graphene}$ heterostructure model system is relevant to a wide range of synthesis conditions in electronics and energy-related applications of $\mathrm{Sb}$ on carbon.

In terms of application potential, we note that trigonally deformed $\mathrm{Sb}$ (like simple cubic Sb) has recently been predicted to feature superior thermoelectrical performance over $\beta-\mathrm{Sb}^{56}$. Given that monolayered $2 \mathrm{D} \beta$-Sb has been predicted to surpass all other pristine $2 \mathrm{D}$ materials in terms of thermoelectric performance ${ }^{27}$, future studies on band structure and electronic properties of the here observed $\beta-\mathrm{Sb}[2-21] /$ cubic $\mathrm{Sb}(001)$ deposits merit consideration.

\section{vdW epitaxy}

So far our data have shown that we have grown 2D Sb/graphene heterostructures, where the $\mathrm{Sb}$ deposits are comprised of two coexisting morphologies, namely few-layer $2 \mathrm{D} \beta-\mathrm{Sb}(001)$ and $1 \mathrm{D}$ nanorods $\beta$-Sb[2-21]/cubic $\mathrm{Sb}(001)$. Importantly, for both these $\mathrm{Sb}$ morphologies Fig. 1a indicated a high degree of directional 
(a)

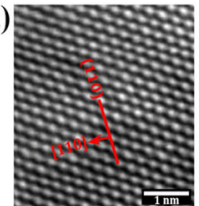

(b)

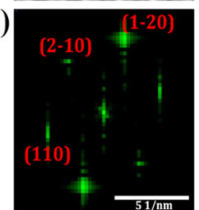

(g)

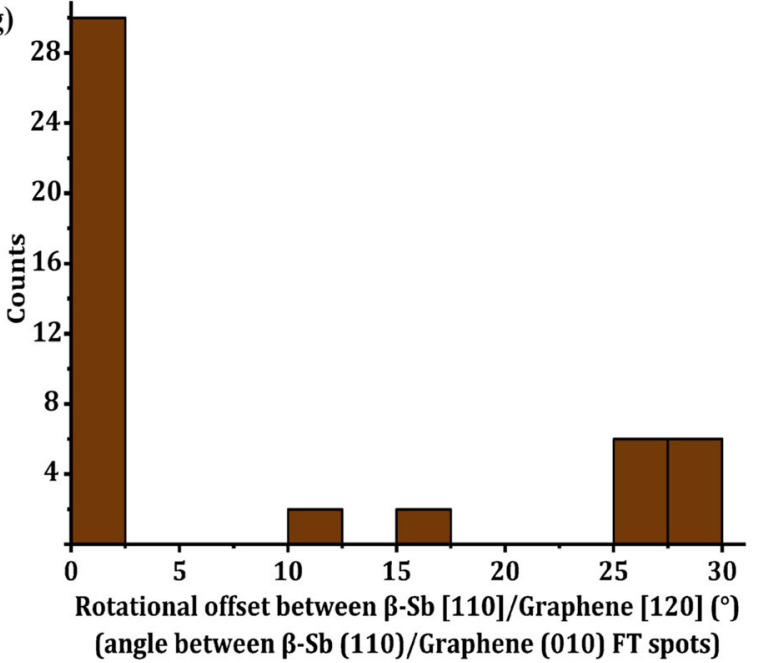

(c)

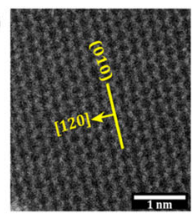

(d)

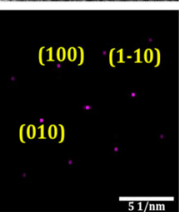

(e)

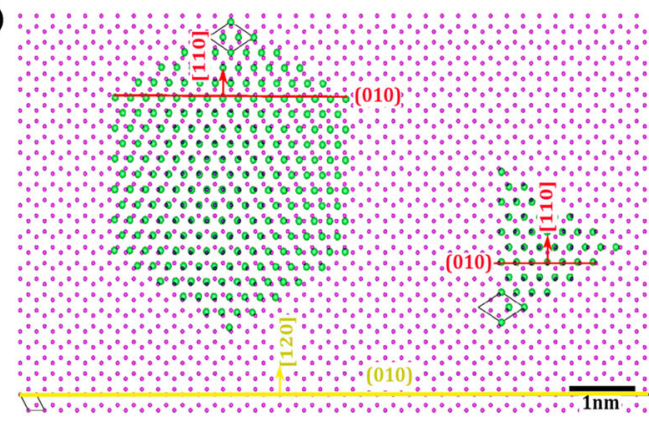

(f)

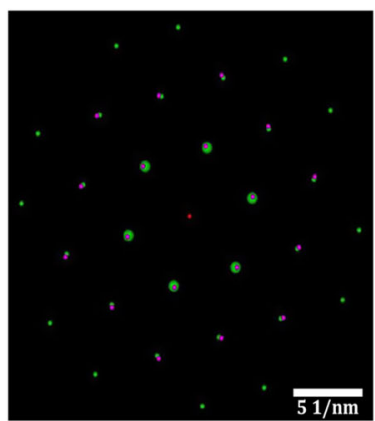

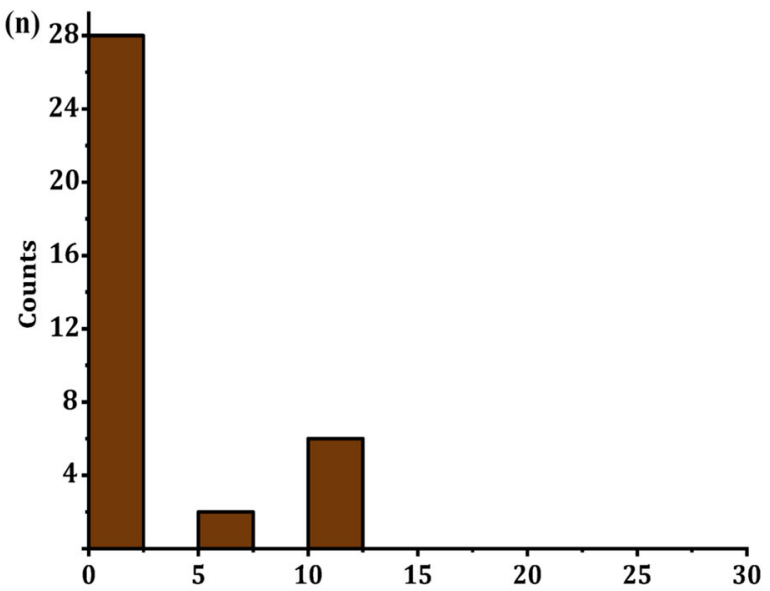

Rotational offset between cubic $\mathrm{Sb}[110] /$ Graphene $[120]\left(^{\circ}\right)$ (angle between cubic $\mathrm{Sb}(110)$ / Graphene (010) FT spots)

(h)

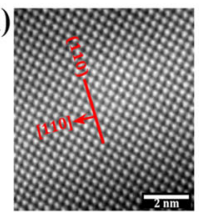

(i)

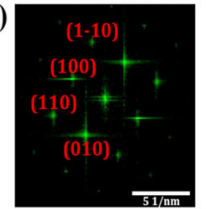

(j)

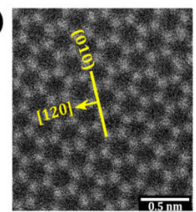

(k)

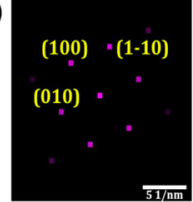

(l)

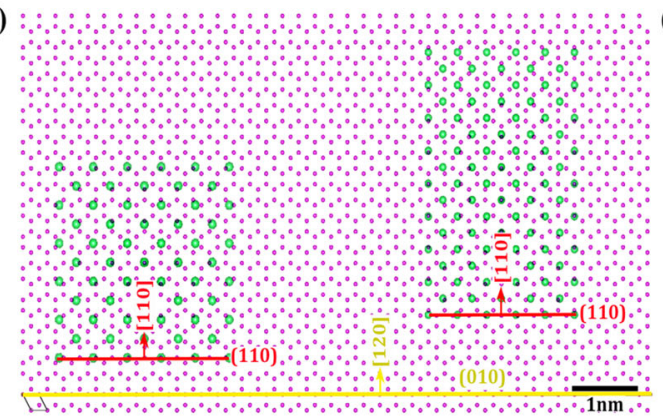

(m)

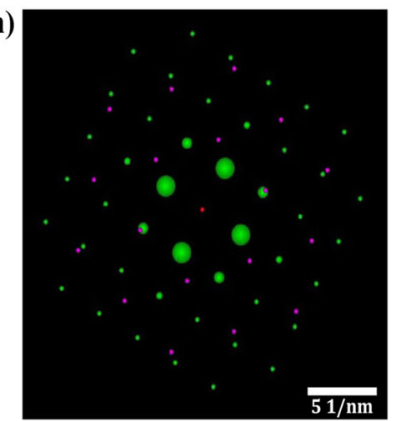

Fig. 3 Epitaxy. a Atomic resolution ADF STEM and $\mathbf{b}$ corresponding FT of a $\beta$-Sb(001) particle on suspended graphene. $\mathbf{c}$ ADF STEM and $\mathbf{d}$ FT of the graphene(001) lattice just adjacent to the particle in a. The FTs are indexed to their corresponding phases and in the ADF images salient planes and directions are highlighted. e Atomic models showing in-plane vdW epitaxial relations derived from the data in a-d, suggesting $\beta$-Sb(001) ||graphene(001)/ $\beta$-Sb[110] |graphene[120], i.e., angle between $\beta$-Sb[110] and graphene[120] $=0^{\circ}$. $\mathbf{f}$ The overlay of simulated FTs corresponding to the models in $\mathbf{e}$. $\mathbf{g}$ Histogram of multiple measurements similar to a-f showing a distribution of (mis)rotation angles between $\beta-\mathrm{Sb}[110]$ and graphene[120] which peaks at $0^{\circ}$, confirming the suggested in-plane vdW epitaxy relation depicted in e to be preferred. $\mathbf{h}-\mathbf{n}$ Corresponding measurements for cubic(001) deposits on suspended graphene, yielding a preferred cubic $\mathrm{Sb}(001) \| \mathrm{graphene}$ (001)/cubic Sb[110]||graphene[120] in-plane vdW epitaxy relation. For epitaxial relations in $\beta$-Sb[2-21] notation see Supplementary Fig. 7. Note that direction vector lengths in $\mathbf{a}, \mathbf{c}, \mathbf{e}, \mathbf{h}, \mathbf{j}$, and $\mathbf{I}$ are not to scale but enlarged for readability.

alignment of their respective domain edges on the monolayer graphene support. Given the vdW nature of the Sb/graphene interface (Fig. 1b), three mechanisms could contribute to such alignment: first is direct rotational vdW epitaxy between the growing $\mathrm{Sb}$ and its graphene support directly underneath ${ }^{72}$. For 2D $\beta$-Sb(001) direct epitaxial relationships with various substrates have been reported, including $\mathrm{WSe}_{2}{ }^{39}$, tellurides ${ }^{32,36}$, mica $^{33}$, and $\mathrm{Ge}^{35}$. Particularly, for $\beta-\mathrm{Sb}(001)$ on graphene prior work has given a mixed picture: Some work $^{38}$ reported rotational vdW epitaxy for $\beta$-Sb/graphene via indirect measurements, while other work observed no such epitaxy ${ }^{31,44,50,51}$. For the 1D nanorod $\beta-\mathrm{Sb}[2-$ $21]$ /cubic $\mathrm{Sb}(001)$, epitaxial effects have to date not been reported $^{31,51}$. Therefore, the question if direct vdW epitaxy is prevalent in the Sb/graphene system remains open. Second, however, complicating elucidation of this question also recently reported remote epitaxy needs consideration in which epitaxy is impressed remotely between a deposit and its underlying substrate through an intermediate 2D layer $^{73}$. In the present work, this would involve interactions between $\mathrm{Sb}$ and the underlying $\mathrm{Cu}$ foils impressed through the graphene monolayer ${ }^{38}$. Notably, in this scenario, the graphene could also be required to act as a diffusion barrier to prevent chemical reactions between $\mathrm{Sb}$ and $\mathrm{Cu}^{74,75}$, thus actually actively facilitating the remote epitaxy. Third, in contrast to the atomic-scale epitaxy, the third possibility 


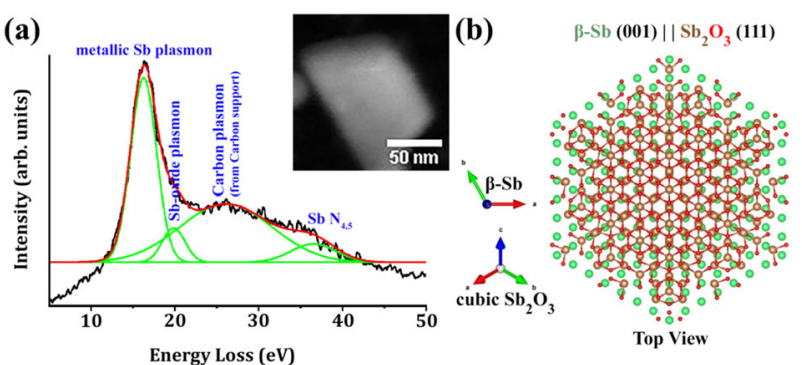

Fig. 4 Oxidation. a VEELS spectrum of the $\beta-S b(001)$ crystal on suspended graphene (on amorphous carbon support) in the ADF STEM in the inset. The spectrum was acquired after $\sim 8$ months ambient air exposure of the sample. The VEELS data are fitted to the components labeled and described in the main text. Measurements on multiple $\beta$ - $\mathrm{Sb}(001)$ and $\beta$-Sb[2-21]/cubic $\mathrm{Sb}(001)$ deposits showed similar results in terms of only low Sb-oxide plasmon signal. Note that the metallic $\mathrm{Sb}$ plasmon has the same energy for $\beta$-Sb(001) and $\beta$-Sb[2-21]/cubic $\mathrm{Sb}(001)$ in our VEELS measurements ${ }^{96}$. b Atomic model of the suggested $\mathrm{Sb}_{2} \mathrm{O}_{3}(111) \| \beta-\mathrm{Sb}(001)$ heterostructure that forms from ambient air exposure on $\beta$-Sb(001) crystals.

involves macroscopic corrugations on the support (e.g., Cu surface steps) that result in alignment via preferred heterogeneous nucleation sites (e.g., at steps) and diffusion directing effects ${ }^{76}$.

From SEM data as in Fig. 1a alone, these three possible causes of the observed $\mathrm{Sb}$ alignments are hard to disentangle: first, direct vdW epitaxy would be readily compatible with the observed lateral length scales of alignment in Fig. 1a as the lateral size of our CVD graphene domains is in the tens of $\mu \mathrm{m}$ range $^{61,62,67}$. Therefore, the field of view in Fig. 1a represents most likely only one single-crystalline graphene domain (although not confirmable by SEM) which could facilitate rotational alignment over the entire field of view. Second, however, graphene-mediated remote epitaxy between $\mathrm{Sb}$ and $\mathrm{Cu}$ is also conceivable for Fig. $1 \mathrm{a}$, since the $\mathrm{Cu}$ grain sizes in our $\mathrm{Cu}$ foils after graphene CVD are in the mm-range ${ }^{61,62}$. Notably, no Cu grain boundary is visible in Fig. $1 a^{62}$, thus confirming a single Cu orientation across the field of view in Fig. 1a. However, as we show in Supplementary Fig. 6, direct deposition of $\mathrm{Sb}$ on $\mathrm{Cu}$ (i.e., without graphene in between) does not show any indications of epitaxial order in the Sb deposits under our conditions ${ }^{74}$. Nevertheless, a graphene-mediated remote epitaxy mechanism between $\mathrm{Sb}$ and $\mathrm{Cu}^{38}$ cannot be excluded based on Fig. 1a. The third possibility, i.e., surface corrugations on the Cu support, could also direct the Sb deposits, although not resolvable in Fig. 1 a.

To disentangle these three possible influences, we investigate in Fig. 3 the relative orientation of $\mathrm{Sb}$ depositions at 150 and $250{ }^{\circ} \mathrm{C}$ directly onto suspended monolayer graphene membranes, i.e., without $\mathrm{Cu}$ foil underneath. In doing so, we exclude any possible indirect influence of $\mathrm{Cu}$ underneath the graphene on the $\mathrm{Sb}$ alignment (i.e., we exclude remote epitaxy and an influence from Cu surface corrugations). In particular, we correlate the STEMderived orientation (via FT analysis) of the lattice of the Sb deposits with the underlying graphene lattice orientation measured adjacent to the $\mathrm{Sb}$ deposit within a few nm distance ${ }^{67}$ for multiple $\mathrm{Sb}$ deposits of both morphologies ( $\beta-\mathrm{Sb}(001)$ : Fig. 3a-g; cubic $\mathrm{Sb}(001)$ : Fig. $3 \mathrm{i}-\mathrm{n} ; \beta-\mathrm{Sb}[2-21]$ : Supplementary Fig. 7). Via plotting histograms of the relative rotational (mis) orientations of the graphene [120] direction (armchair direction) and prominent orientations in the two respective Sb lattices ( $\beta$-Sb [110]: Fig. 3g; cubic Sb [110]: Fig. 3n; $\beta-S b[2-21]$ : Supplementary Fig. 7), we find clear peaks in the (mis)orientation distributions for both $\mathrm{Sb}$ phases. This unambiguously suggests direct epitaxy effects to be present between the graphene and both Sb phases. In particular $2 \mathrm{D} \beta$-Sb shows a preferred misorientation of $\sim 0^{\circ}$ between the graphene [120] and the $\beta$-Sb [110] in-plane directions (i.e., $\beta$-Sb [110]\|graphene[120]), as shown in the model in Fig. 3e. In addition, a secondary, less prominently preferred misorientation appears at an offset of $\sim 30^{\circ}$ for $\beta$-Sb [110] and graphene [120] in Fig. 3e. For cubic Sb, we find a single preferred misorientation of $\sim 0^{\circ}$ between the graphene [120] and cubic Sb [110] directions (i.e., cubic Sb[110]||graphene[120]), as depicted in the model in Fig. 3l. Notably, since the long axis direction of the cubic Sb rods is [110] (Fig. 1), this implies that the rods' long axes are aligned along the graphene [120] direction. Notably, this preferential alignment may be intertwined with symmetry breaking of the cubic $\mathrm{Sb}(001)$ toward anisotropic rod formation.

To cross-check these STEM-derived rotational vdW epitaxy relations via the SEM data in Fig. 1a, we label the salient directions by colored arrows in Fig. 1a: we first assign the long axis of the cubic Sb rods to cubic Sb [110] based on Fig. 1e-g (red arrow). Based on cubic Sb[110] |graphene[120], this direction then coincides with graphene [120] direction (yellow arrow). Thereby it becomes apparent that for most of the $\beta$-Sb triangles in Fig. 1a one triangle edge (red arrow) coincides with the graphene[120] direction. This is exactly as expected from the $\beta$-Sb [110]|| graphene[120] relation and from the observation that one edge direction of the triangles is typically $\beta$-Sb [110], as inferred in Fig. 1c, d. Consequently, STEM and SEM data consistently suggest direct Sb/graphene vdW epitaxy for both $\beta$-Sb and cubic Sb with the preferred overall relations $\beta$ - $\mathrm{Sb}(001) \|$ graphene $(001) / \beta-\mathrm{Sb}$ [110] |graphene[120] and cubic $\mathrm{Sb}(001) \|$ graphene(001)/cubic $\mathrm{Sb}[110]$ ||graphene[120], respectively. (For epitaxial relations in $\beta$-Sb[2-21] notation see Supplementary Fig. 7).

Prior work has investigated possible epitaxy between Sb polymorphs and graphene (and graphite) with mixed results: early work did not find evidence for epitaxy in $\beta$-Sb/graphite (but had only limited statistics measured $)^{51}$. Also, recent other studies did not observe epitaxy in $\beta$-Sb/graphene ${ }^{31,44}$. In contrast, another recent study of $\beta$-Sb and Cu-supported graphene suggested epitaxy for $\beta-S b / g r a p h e n e$ to exist based on indirect measurements, identifying two preferred orientations of (in our notation) $0^{\circ}$ and $30^{\circ}$ offset between $\beta$-Sb [110] and graphene $[120]^{38}$. This is in good agreement with our findings in Fig. $3 \mathrm{~g}$ which are based on direct observations of the $\beta$-Sb/graphene interface. For $\beta-S b[2-21] /$ cubic $\mathrm{Sb}(001)$ on graphite no evidence for epitaxy has been reported prior ${ }^{31,51}$. In contrast, we here find strong evidence also for rotational vdW epitaxy in the $\beta-S b[2-21] /$ cubic $\mathrm{Sb}(001)$ / graphene system. Combined, our observations show that vdW epitaxy can be enforced on 2D and 1D Sb deposits on graphene.

\section{Oxidation susceptibility}

After having identified the nature of our Sb deposits and their relation to the graphene support, we turn to the oxidation susceptibility of our Sb deposits. Oxidation susceptibility is of significant importance in terms of processing and applications. In addition, 2D Sb oxides are beginning to attract research interest in their own right ${ }^{64,77,78}$. While Raman spectroscopy in Fig. 1b did not suggest significant Sb-oxide presence in our samples, close inspection of the $\beta$-Sb(001) FT in Fig. 1d reveals another, weaker intensity set of spots of sixfold symmetry at lower k-vectors (indexed "*" at $\sim 0.4 \mathrm{~nm}$ ) than the sixfold (110) $\beta-\mathrm{Sb}(001)$ spot family which is indexed in the FT. These weak inner spots may be identified with the presence of cubic $\mathrm{Sb}_{2} \mathrm{O}_{3}$ viewed along the [111] zone axis ${ }^{64}$ (i.e., cubic $\mathrm{Sb}_{2} \mathrm{O}_{3}(111)$, see Supplementary Figs. 2 and 3 ). This poses the question whether our $\beta$-Sb deposits are partly and/or superficially oxidized during sample storage in ambient air. Some prior work has reported stability of antimonene against oxidation in the ambient conditions ${ }^{33,35,36,42,43,46,79}$ but other work has suggested thin antimony oxide present around $\mathrm{Sb}$ structures to be also prevalent ${ }^{22,23,26,38,80-82}$. Notably, for the $\beta-S b$ [2-21]/cubic $\mathrm{Sb}(001)$ deposits no signs of additional crystalline oxides are found in (S)TEM or FT data. 

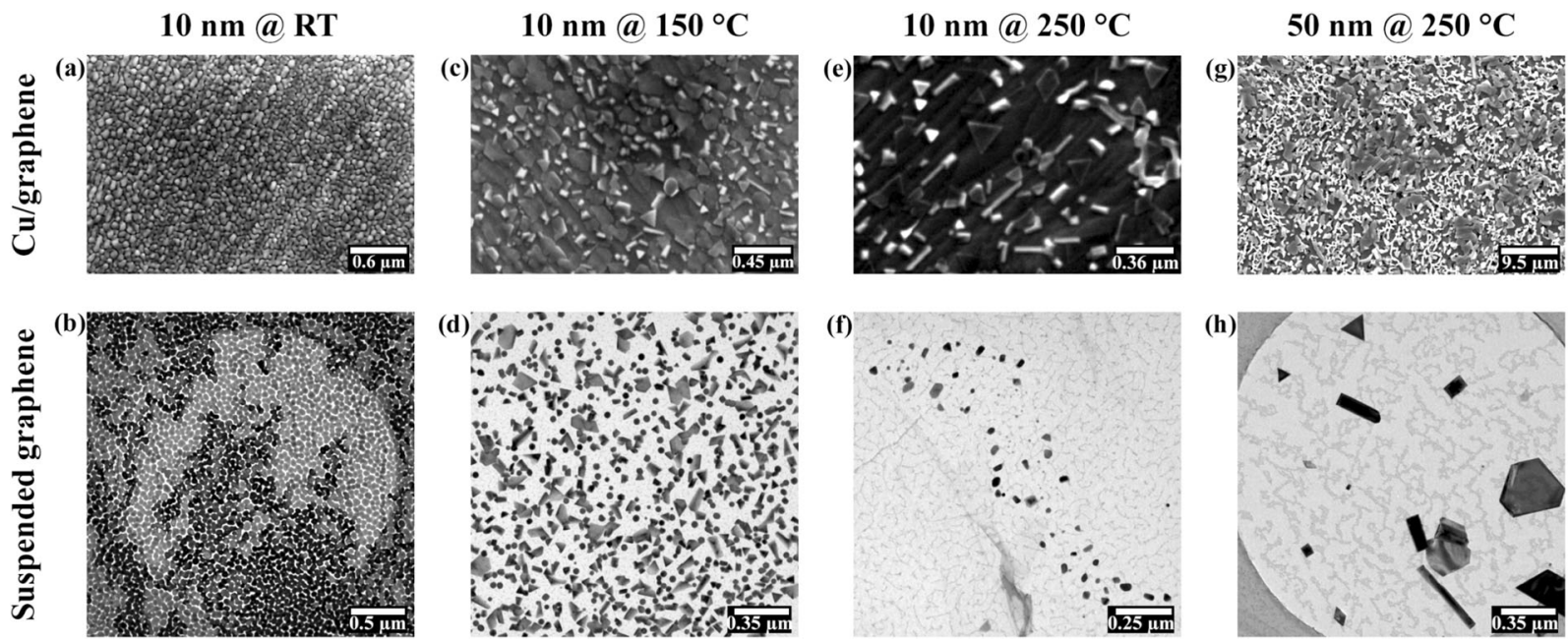

Fig. 5 Growth parameter space. Sb deposits on Cu-supported graphene (SEM in a, $\mathbf{c}, \mathbf{e}, \mathbf{g}$ ) and suspended graphene (BF-TEM in b, d, f, $\mathbf{h}$ ) deposited at nominal thicknesses of $10 \mathrm{~nm}$ at $\mathrm{RT}(\mathbf{a}, \mathbf{b}), 150^{\circ} \mathrm{C}(\mathbf{c}, \mathbf{d})$, and $250^{\circ} \mathrm{C}(\mathbf{e}, \mathbf{f})$ and $50 \mathrm{~nm}$ at $250{ }^{\circ} \mathrm{C}(\mathbf{g}$, h), respectively. e is replotted from Fig. 1 a.

To investigate possible oxidation effects for our Sb deposits in a localized fashion, we use chemical identification via electron energy loss spectroscopy (EELS). In EELS of Sb/Sb-oxide mixtures, compositional analysis based on the commonly used EELS core loss regions is however difficult since the core loss $\mathrm{Sb} \mathrm{M}_{4,5}$ edge at $\sim 528 \mathrm{eV}$ (which follows a delayed maxima fashion) is very close/ partially overlapping the O K-edge at $\sim 532 \mathrm{eV}$ (Supplementary Fig. $8)^{83,84}$. An alternative approach is investigating the valence EELS (VEELS) low loss region in which a sharp bulk plasmon peak at $\sim 16.8 \mathrm{eV}$ is related to metallic $\mathrm{Sb}^{85}$, while the plasmon peak shifts for $\mathrm{Sb}$ oxides to a distinctly higher energy of $\sim 22 \mathrm{eV}^{83}$. For VEELS, however, also the graphene support (and, if present, amorphous carbon TEM grid membrane) has to be considered with plasmon signatures at $\sim 27 \mathrm{eV}^{86}$.

In Fig. 4a, a typical VEELS spectrum acquired from a flat 2D $\beta$-Sb crystal (inset) is shown. We note that this particular Sb deposit was characterized by VEELS after $\sim 8$ months of ambient air storage, thus allowing us to probe long-term resilience against oxidation. We find that a sharp metallic Sb VEELS peak is dominating the fitted VEELS spectrum with only a small contribution of the Sboxide component present even after the long-term air exposure. VEELS data for $\beta-S b[2-21] / c u b i c ~ S b(001)$ deposits show similar results of only low Sb-oxide signal. The VEELS findings are thereby in agreement with the Raman data in Fig. 1b that suggested metallic $\mathrm{Sb}$ to be dominant in our deposits. The VEELS results however suggest the possibility of a very thin superficial oxide layer (which might be below the detection limit for Raman). This further implies for the $\beta-\mathrm{Sb}(001)$ that the inner reflections (labeled "**) in the FT in Fig. 1d may be indeed related to a very thin crystalline cubic $\mathrm{Sb}_{2} \mathrm{O}_{3}(111)$ top layer on the elemental 2D $\beta$-Sb (001) crystal (where reflection "** in Fig. 1d corresponds to the (2-20) reflection in $\mathrm{Sb}_{2} \mathrm{O}_{3}$, see also Supplementary Figs. 2 and 3). This suggests the possibility of intrinsic Sb-oxide/Sb/graphene heterostructure formation from simple ambient air exposure. In particular, whenever present, the sixfold $\mathrm{Sb}_{2} \mathrm{O}_{3}$ (2-20) reflection family consistently has a rotational misorientation of $\sim 30^{\circ}$ with the sixfold $\beta$-Sb (110) reflection family (as in Fig. $1 d$ ). This indicates an epitaxial relation $\mathrm{Sb}_{2} \mathrm{O}_{3}(111)\left\|\beta-\mathrm{Sb}(001) / \mathrm{Sb}_{2} \mathrm{O}_{3}[2-20]\right\| \beta-\mathrm{Sb}[110]$ for the $\mathrm{Sb}$-oxide/Sb interface. See Fig. $4 \mathrm{~b}$ for an atomic model of the suggested $\mathrm{Sb}$-oxide/Sb heterostructure ( $\mathrm{Sb}$ oxide/Sb/graphene). Unfortunately, the top regions in the $\beta$-Sb flakes in our crosssection (S)TEM data are all not well enough resolved (due to $\mathrm{Pt} / \mathrm{C}$ protection layers) to finally fully confirm the suggested presence of this ultrathin epitaxial $\mathrm{Sb}_{2} \mathrm{O}_{3}$ top layer. We note however that prior X-ray photoelectron spectroscopy measurements of 2D Sb oxidation found oxide stoichiometries consistent with the here suggested crystalline $\mathrm{Sb}_{2} \mathrm{O}_{3}$ phase ${ }^{81}$, and that our core loss EELS in Supplementary Fig. 8 also is best matched with mixed $\mathrm{Sb}$ and $\mathrm{Sb}_{2} \mathrm{O}_{3}$ stoichiometry. No such crystalline overlayers are suggested from our data for the $\beta-\mathrm{Sb}[2-21] /$ cubic $\mathrm{Sb}(001)$, albeit a thin amorphous Sb-oxide overlayer is suggested to be also present based on the presence of the minor Sb-oxide signals in VEELS. Combined, our microscopic and spectroscopic data show that while our $\mathrm{Sb}$ deposits are overall well resilient against environmental bulk oxidation, the possibility of superficial oxidation in ambient air still requires consideration, in particular since for $\beta$-Sb (001) deposits the formation of an epitaxial $\mathrm{Sb}_{2} \mathrm{O}_{3}(111)$ oxide overlayer is inferred from our data.

\section{Growth parameter space}

Finally, we examine the wider parameter space of our Sb PVD. Figure 5 compares $\mathrm{Sb}$ deposition results of nominally $10 \mathrm{~nm} \mathrm{Sb}$ (regulated via a coexposed (nonheated) QMB) as function of substrate temperature from RT to $250^{\circ} \mathrm{C}$ onto CVD graphenecovered $\mathrm{Cu}$ foils (Fig. 5a, C, e, SEM) as well as directly onto suspended graphene membranes (no Cu underneath, Fig. 5b, d, f, TEM). Figure 5 shows that the Sb deposit morphology drastically changes between RT and elevated temperature $\left(150^{\circ} \mathrm{C}, 250^{\circ} \mathrm{C}\right)$ depositions: for RT depositions (Fig. 5a, b) merged (truncated) (semi-)spherical features dominate. High-resolution STEM in Supplementary Fig. 9 shows that these RT-deposited (truncated) (semi-)spheres are amorphous. For $150^{\circ} \mathrm{C}$ depositions (Fig. $5 \mathrm{C}, \mathrm{d}$ ) the above-described triangular/hexagonal-shaped $2 \mathrm{D} \beta$-Sb and rod-shaped cubic Sb crystals along with few (semi-)spherical Sb deposits are found. Among the 2D $\beta$-Sb deposits the hexagonal base shape is more prevalent. For $250^{\circ} \mathrm{C}$ depositions (Fig. 5e, f), practically only triangular/hexagonal-shaped $2 \mathrm{D} \beta$-Sb and rodshaped cubic Sb are found, whereby now among the 2D $\beta$-Sb triangles dominate. Notably, for neither $150^{\circ} \mathrm{C}$ nor $250^{\circ} \mathrm{C}$ we find evidence for an underlying continuous $\mathrm{Sb}$ layer on neither $\mathrm{Cu}$ supported nor freestanding graphene, the former in contrast to the prior literature ${ }^{38}$. Besides deposit morphology, also coverage and retained $\mathrm{Sb}$ amount of the nominally $10 \mathrm{~nm}$ Sb deposits is strongly influenced by substrate temperature during $\mathrm{Sb}$ deposition and, notably, also strongly dependent on substrate type. In particular, Sb coverage and retained Sb amount strongly decrease with increasing substrate temperature. For RT depositions, a homogeneous coverage close to $100 \%$ is achieved on both $\mathrm{Cu}$ supported and freestanding graphene in Fig. $5 a, b$ and for RT 
samples good agreement between nominal $10 \mathrm{~nm}$ thickness and AFM-calibrated average deposit thickness was found. In comparison, the coverage for 150 and $250{ }^{\circ} \mathrm{C}$ depositions decreases, whereby the coverage decrease with substrate temperature is even more prominent on the freestanding graphene $\left(150^{\circ} \mathrm{C}: \sim 40 \%, 250^{\circ} \mathrm{C}:<5 \%\right)$ than on the $\mathrm{Cu}$-supported graphene $\left(150^{\circ} \mathrm{C}: \sim 40 \%, 250^{\circ} \mathrm{C}: \sim 20 \%\right)$. For $\mathrm{Sb}$ deposits with an average thickness of $21 \pm 14 \mathrm{~nm}$ at $250^{\circ} \mathrm{C}$ (see above), this equates to a reduction in $\mathrm{Sb}$ amount deposited from RT to $250^{\circ} \mathrm{C}$ of $\sim 50 \%$ on Cu-supported graphene and of $\sim 90 \%$ on freestanding graphene, respectively. Notably, also the size of individual deposits of $\mathrm{Cu}$ is significantly larger than on the suspended graphene, best seen in the $250^{\circ} \mathrm{C}$ depositions (Fig. 5e, f). Combined, this suggests a key influence of temperature dependent desorption processes on Sb nanostructure growth $^{35,37,47-51}$. In particular, the balance of Sb adsorption flux $\left(F_{\mathrm{Sb}, \text { ad }}\right)$ from the evaporation source onto the graphene substrate and a substrate temperature-dependent $\mathrm{Sb}$ desorption flux from the graphene substrate into vacuum $\left(F_{\mathrm{Sb}, \mathrm{de}}(T)\right)$ is key: the observed $\mathrm{Sb}$ morphologies imply that at $\mathrm{RT} F_{\mathrm{Sb}, \mathrm{ad}}$ » $F_{\mathrm{Sb} \text {,de }}(\mathrm{RT})$ results in strong deposition, while the low temperature hinders crystallization of the resulting $\mathrm{Sb}$ deposits (possibly via incomplete fragmentation of physisorbed $\mathrm{Sb}_{4}$ species which are the preferred arriving $\mathrm{Sb}$ vapor species ${ }^{51}$ ). This leads to the observed fully covering amorphous $\mathrm{Sb}$ deposits at RT. Increasing substrate temperature leads to a strong increase in $F_{S b, d e}\left(150-250^{\circ} \mathrm{C}\right)$, reducing the net retained amount of $\mathrm{Sb}$ at higher substrate temperature. In turn, the higher substrate temperatures facilitate crystallization of the retained Sb deposits (possibly via thermally activated fragmentation of surface-bound $\mathrm{Sb}$ species ${ }^{51}$ and thus increased $\mathrm{Sb}$ rearrangement). Thereby we grow crystalline $\mathrm{Sb}$ deposits with an onset temperature of crystallization of $\sim 150^{\circ} \mathrm{C}$. The observation that this temperature dependence is more pronounced on freestanding graphene membranes as compared to Cu-foil supported graphene, we suggest to be related either to intrinsic substrate effects whereby the Cu surface states underneath the graphene modify, e.g., sticking coefficients to the $\mathrm{Sb}$ flux (akin to Cu supports modifying the surface properties of graphene in liquid wetting ${ }^{87,88}$ ) or to a different local temperature profile on Cu-foil supported graphene vs. suspended graphene membranes due to the macroscopic thickness $25 \mu \mathrm{m}$ Cu foils. In the latter scenario, the Cu foil acts as an effective heat sink for the additional energy arriving with the incoming $\mathrm{Sb}$ flux $F_{\mathrm{Sb}, \text { ad }}$ compared to the vacuum-suspended monolayer graphene membranes (and, if present, thin amorphous carbon support), thus resulting in a (slightly) lower actual local substrate temperatures on the $\mathrm{Cu}$ supported graphene.

The here observed temperature dependence of $\mathrm{Sb}$ deposit morphology, crystallization onset, and retained Sb amount is in good agreement with the prior literature ${ }^{35,37,47-51}$. Beyond this, our results confirm that not only the directly supporting growth substrate (here, monolayer graphene) but also the supporting material underneath (here, Cu vs. vacuum) can strongly influence $\mathrm{Sb}$ nanostructure growth results ${ }^{38}$. This is important to consider when designing $\mathrm{Sb} 2 \mathrm{D} / 2 \mathrm{D}$ heterostructure stacks. Finally, in Fig. $5 \mathrm{~g}$, $\mathrm{h}$, we show that the here derived understanding of the balance of adsorption, nucleation, desorption, and sub-support can also be advantageously employed to engineer larger $\mathrm{Sb}$ deposits of high crystalline quality. Figure $5 \mathrm{~g}$, h shows deposition of nominally $\sim 50 \mathrm{~nm} \mathrm{Sb}$ at $250^{\circ} \mathrm{C}$ on Cu-supported (Fig. $5 \mathrm{~g}$ ) and freely suspended graphene (Fig. $5 \mathrm{~h}$ ). On the Cu-supported graphene, increasing the deposited Sb amount led not to laterally larger $\mathrm{Sb}$ domains but to the onset of undesired $3 \mathrm{D} \mathrm{Sb}$ overgrowth (Fig. $5 \mathrm{~g}$ ). In contrast, the relatively higher desorption on the suspended graphene enabled a lower $\mathrm{Sb}$ nucleation density and consequently a desired larger lateral growth of remaining $\mathrm{Sb}$ crystallites (Fig. $5 \mathrm{~h}$, since presumably desorption probability decreases with increasing deposit radius). Thereby by adjusting the substrate underneath the actual graphene support, we obtain a lateral size of $>400 \mathrm{~nm}$ diagonal for $\beta-\mathrm{Sb}(001)$ and $>500 \mathrm{~nm}$ long axis for $\beta$-Sb[2-21]/cubic Sb(001) deposits, respectively. This is an improvement not only over the undesired 3D Sb overgrowth from Cu/graphene supported $250^{\circ} \mathrm{C} / 50 \mathrm{~nm}$ but also an improvement of factor $\sim 2$ compared to the $250^{\circ} \mathrm{C} / 10 \mathrm{~nm} \mathrm{Cu} /$ graphene-supported deposits. This introduces the substrate underneath the direct 2D support as an important parameter to consider in 2D Sb deposition.

In summary, using high-resolution STEM, we elucidate the structural relations in 2D Sb/graphene heterostructures which we present as a model system for 2D Sb's use in electronics and energy applications. We find two Sb morphologies to coexist under optimized deposition conditions: few-layer 2D $\beta$-Sb(001)\| graphene(001) and 1D Sb which can be matched to both $\mathrm{Sb}[2-$ $21] \perp$ graphene $(001)$ and cubic $\mathrm{Sb}(001) \|$ graphene(001). Notably, both morphologies exhibit direct in-plane rotational vdW epitaxy with the graphene support. Both morphologies are stable against ambient air oxidation even for prolonged storage, albeit superficial surface $\mathrm{Sb}$-oxide formation is found. Notably, for $\beta$-Sb(001) growth of an epitaxial $\mathrm{Sb}_{2} \mathrm{O}_{3}(111) \| \beta-\mathrm{Sb}(001)$ overlayer is suggested from our data. While exact $\mathrm{Sb}$ growth results depend on growth parameters such as temperature, importantly also the nature of the support under the direct graphene support is found to have a key influence on Sb growth. Combined, our findings explore at high resolution the structural diversity in scalably fabricated $2 \mathrm{D} \mathrm{Sb}$ and in 2D Sb/graphene heterostructures.

\section{METHODS}

\section{Growth of 2D Sb and grapheme}

PVD of Sb employed a commercial thermal evaporation system (MANTIS deposition system QUBE series) with a base pressure of $4 \times 10^{-5}$ mbar. For PVD, Sb powder (Goodfellow, 99.999\% purity, average particle size $150 \mu \mathrm{m}$ ) was loaded into a W boat, which was heated resistively to sublime the Sb. Phase diagrams ${ }^{89}$ of $\mathrm{W}$ and $\mathrm{Sb}$ were cross-checked to ensure that no undesired intermetallics are formed during evaporation. Samples were loaded upside down over the evaporation source and behind a manual shutter. The sample table was electrically heated to a desired substrate temperature, where RT (i.e., nonheated), 150 and $250^{\circ} \mathrm{C}$ were employed in this study. The Sb evaporation flux and nominally deposited thickness were monitored in situ using a nonheated QMB. The nominal Sb thickness QMB measurement was calibrated by evaporation of selected Sb films over partially masked Si wafers (thickness monitors) at RT to measure Sb film thickness over film edges by AFM. Note that the nominal thicknesses quoted in this study refer to the measured thickness values obtained from the nonheated QMB and from these Si wafer calibration depositions at RT. As discussed in the exploration of the parameter space of Sb PVD in the main text, actual retained $\mathrm{Sb}$ thicknesses can strongly reduce as a function of increasing substrate temperature and also type of substrate type via desorption effects.

Substrates for $\mathrm{Sb}$ deposition were CVD $\left(960{ }^{\circ} \mathrm{C}\right.$ in $\mathrm{CH}_{4} / \mathrm{H}_{2} / \mathrm{Ar}$ at $\sim 12$ mbar) polycrystalline (grain size tens of $\mu \mathrm{m}$ ) monolayer graphene films remaining on their $25 \mu \mathrm{m}$ thick Cu-foil catalysts ${ }^{61,62}$ as well as CVD graphene films suspended as freestanding monolayer membranes over the regular hole arrays in an amorphous carbon film of a TEM grid (Quantifoil), i.e., no Cu underneath ${ }^{63}$. For graphene-free reference also $\mathrm{Cu}$ foils without graphene were prepared as substrates by annealing at $960^{\circ} \mathrm{C}$ in $12 \mathrm{mbar} \mathrm{H}_{2}$ without $\mathrm{CH}_{4}$.

Samples were handled and stored in ambient atmosphere between ex situ graphene and 2D Sb preparation steps and STEM measurements, resulting in adventitious hydrocarbon contaminations on the graphene membranes (Figs. 1 and 5) ${ }^{59}$, which are however typical and relevant for scalable 2D heterostructure processing ${ }^{68}$.

\section{Microscopic and spectroscopic measurements}

The SEM employed a FEI Quanta 250 FEG SEM. TEM studies including BF imaging, SAED, (V)EELS, and energy dispersive X-ray spectroscopy (EDX, confirming the Sb purity) were performed on a FEI TECNAI F20 at $60 \mathrm{kV}$ electron acceleration voltage. STEM studies were performed in an aberration-corrected Nion UltraSTEM 100 at $60 \mathrm{kV}$ electron acceleration 
voltage and in (high angle) ADF ((HA)ADF) mode ( $80-200 \mathrm{mrad})^{60}$. STEM and BF-TEM image contrast was autoset via Image $J^{90}$ software image by image. Correlative AFM-SEM studies employed a GETec AFSEM module installed in a FEI Quanta 600F SEM ${ }^{71}$. Conventional AFM studies employed a NT MDT NTEGRA Spectra in tapping mode ${ }^{91}$. AFM analysis employed Gwyddion software ${ }^{92}$. Raman spectroscopy employed a Horiba LabRAM at $532 \mathrm{~nm}$ laser excitation wavelength. Cross sections for TEM/STEM of $\mathrm{Cu} /$ graphene/Sb stacks were cut by FIB processing in a FEI Quanta 3D FEG. A protective $\mathrm{C}$ and $\mathrm{Pt}$ bilayer was deposited in FIB locally onto the region of interest prior to FIB cutting.

\section{Phase and epitaxy analysis of Sb deposits}

Phase analysis of (S)TEM data employed primarily FT/SAED pattern simulation using Highscore Plus/Pdf4+ software (ICDD Pdf4+ 2020 RDB: software version: 4.20.0.1. Database version: 4.2001.) for manual matching of measured and simulated FT/SAED patterns. In addition, also automated phase identification of measured FT/SAED data was performed using JEMS software. Structure visualization was done by Vesta ${ }^{93}$ software. In particular, the following structural database entries were found to best fit our measured FT and SAED data (Pdf4+ code/Inorganic Crystal Structure Database ICSD collection code/literature reference): $\beta$-Sb: 04-14-2871/ 55402/ref. ${ }^{94}$; simple cubic Sb: 04-13-3319/651499/ref. ${ }^{47}$; and $\mathrm{Sb}_{2} \mathrm{O}_{3}: 00-$ $042-1466 / 1944 /$ ref. $^{95}$. Notably, we checked additionally 45 other Sb and 61 other Sb-oxide entries from the ICDD Pdf4+ database which consistently gave worse matches to experimental data.

Note that $\beta$-Sb $(A 7, R-3 m, 166)$ is often described in the literature not only with hexagonal axis (as here) but also with rhombohedral axis ${ }^{33,38,51}$ Therefore, numerical (hkl) and [uvw] values need consideration of selected hexagonal or rhombohedral axis system, when comparing between reports. Likewise, within the hexagonal axis system some literature uses a $a, b$ base vector inner angle of $120^{\circ}$ (as here), while other literature uses $a, b$ base vector inner angle of $60^{\circ 31,33,38,51}$. Again therefore comparison of numerical (hkl) and [uvw] values must consider the selected axis system. To avoid ambiguity the here used axis are typically plotted alongside the atomic models throughout the manuscript.

We calculated the average equivalent feature sizes (see main text) for the $\mathrm{Sb}$ deposits from their base areas (computed by Image $\mathrm{J}^{90}$ ) in SEM and AFM data as follows: due to the different asymmetries between the characteristic base shapes for 2D triangular/hexagonal $\beta-S b(001)$ and $1 D$ rod-like $\beta$-Sb[2-21]/cubic $\mathrm{Sb}(001)$, we recalculate a characteristic feature size assuming a square base shape for both phases and defining the side length of this square as the equivalent feature size.

\section{DATA AVAILABILITY}

The authors declare that the data supporting the findings of this study are available within the paper and its supplementary information files.

Received: 7 January 2021; Accepted: 1 April 2021; Published online: 14 May 2021

\section{REFERENCES}

1. Pumera, M. \& Sofer, Z. 2D monoelemental arsenene, antimonene, and bismuthene: beyond black phosphorus. Adv. Mater. 29, 1605299 (2017).

2. Zhang, $S$. et al. Recent progress in $2 D$ group-VA semiconductors: from theory to experiment. Chem. Soc. Rev. 47, 982-1021 (2018).

3. Ersan, F. et al. Two-dimensional pnictogens: a review of recent progresses and future research directions. Appl. Phys. Rev. 6, 021308 (2019).

4. Zhao, A. et al. Review of 2D group VA material-based heterostructures. J. Phys. Appl. Phys. 53, 293002 (2020).

5. Ares, P., Palacios, J. J., Abellán, G., Gómez-Herrero, J. \& Zamora, F. Recent progress on antimonene: a new bidimensional material. Adv. Mater. 30, 1703771 (2018).

6. Wang, X., Song, J. \& Qu, J. Antimonene: from experimental preparation to practical application. Angew. Chem. Int. Ed. 58, 1574-1584 (2019).

7. Zhang, S., Yan, Z., Li, Y., Chen, Z. \& Zeng, H. Atomically thin arsenene and antimonene: semimetal-semiconductor and indirect-direct band-gap transitions. Angew. Chem. 127, 3155-3158 (2015).

8. Wang, G., Pandey, R. \& Karna, S. P. Atomically thin group V elemental films: theoretical investigations of antimonene allotropes. ACS Appl. Mater. Interfaces 7, 11490-11496 (2015).
9. Pizzi, G. et al. Performance of arsenene and antimonene double-gate MOSFETs from first principles. Nat. Commun. 7, 12585 (2016).

10. Zhao, M., Zhang, X. \& Li, L. Strain-driven band inversion and topological aspects in Antimonene. Sci. Rep. 5, 16108 (2015).

11. Qian, J. et al. High capacity Na-storage and superior cyclability of nanocomposite $\mathrm{Sb} / \mathrm{C}$ anode for Na-ion batteries. Chem. Commun. 48, 7070-7072 (2012).

12. Zhou, X. et al. Sb nanoparticles decorated N-rich carbon nanosheets as anode materials for sodium ion batteries with superior rate capability and long cycling stability. Chem. Commun. 50, 12888-12891 (2014).

13. Hu, L. et al. A chemically coupled antimony/multilayer graphene hybrid as a highperformance anode for sodium-ion batteries. Chem. Mater. 27, 8138-8145 (2015).

14. Cheng, Y. et al. Nanostructured carbon/antimony composites as anode materials for lithium-ion batteries with long life. Chem. Asian J. 11, 2173-2180 (2016).

15. Liu, X. et al. Enhancing the anode performance of antimony through nitrogendoped carbon and carbon nanotubes. J. Phys. Chem. C 120, 3214-3220 (2016).

16. $\mathrm{Gu}, \mathrm{J}$. et al. Liquid-phase exfoliated metallic antimony nanosheets toward high volumetric sodium storage. Adv. Energy Mater. 7, 1700447 (2017).

17. Cui, C. et al. Antimony nanorod encapsulated in cross-linked carbon for highperformance sodium ion battery anodes. Nano Lett. 19, 538-544 (2018).

18. Tian, W. et al. Few-layer antimonene: anisotropic expansion and reversible crystalline-phase evolution enable large-capacity and long-life $\mathrm{Na}$-ion batteries. ACS Nano 12, 1887-1893 (2018).

19. Wang, H., Wu, X., Qi, X., Zhao, W. \& Ju, Z. Sb nanoparticles encapsulated in 3D porous carbon as anode material for lithium-ion and potassium-ion batteries. Mater. Res. Bull. 103, 32-37 (2018).

20. Zhang, X. et al. Metallic Sb nanoparticles embedded in carbon nanosheets as anode material for lithium ion batteries with superior rate capability and long cycling stability. Electrochim. Acta 283, 1689-1694 (2018).

21. Walter, M., Erni, R. \& Kovalenko, M. V. Inexpensive antimony nanocrystals and their composites with red phosphorus as high-performance anode materials for Na-ion batteries. Sci. Rep. 5, 8418 (2015).

22. Li, F. et al. Unlocking the electrocatalytic activity of antimony for $\mathrm{CO} 2$ reduction by two-dimensional engineering of the bulk material. Angew. Chem. 129, 14910-14914 (2017).

23. Gusmão, R., Sofer, Z., Bouša, D. \& Pumera, M. Pnictogen (As, Sb, Bi) nanosheets for electrochemical applications are produced by shear exfoliation using kitchen blenders. Angew. Chem. 129, 14609-14614 (2017).

24. $\mathrm{Yu}, \mathrm{X}$. et al. Emerging 2D pnictogens for catalytic applications: status and challenges. J. Mater. Chem. A 8, 12887-12927 (2020).

25. Martínez-Periñán, E. et al. Antimonene: a novel 2D nanomaterial for supercapacitor applications. Adv. Energy Mater. 8, 1702606 (2018).

26. Wang, X. et al. Bandgap-tunable preparation of smooth and large two-dimensional antimonene. Angew. Chem. 130, 8804-8809 (2018).

27. Chen, K.-X. et al. Excellent thermoelectric performance predicted in twodimensional buckled antimonene: a first-principles study. J. Phys. Chem. C 121 13035-13042 (2017).

28. Zhang, S. et al. Semiconducting group 15 monolayers: a broad range of band gaps and high carrier mobilities. Angew. Chem. 128, 1698-1701 (2016).

29. Aktürk, O. Ü., Özçelik, V. O. \& Ciraci, S. Single-layer crystalline phases of antimony: antimonenes. Phys. Rev. B 91, 235446 (2015).

30. Hogan, C. et al. Temperature driven phase transition at the antimonene/Bi2Se3 van der Waals heterostructure. ACS Nano 13, 10481-10489 (2019).

31. Fortin-Deschênes, $M$. et al. Pnictogens allotropy and phase transformation during van der Waals growth. Nano Lett. 20, 8258-8266 (2020).

32. Lei, T. et al. Electronic structure of antimonene grown on Sb2Te3 (111) and Bi2Te3 substrates. J. Appl. Phys. 119, 015302 (2016).

33. Ji, J. et al. Two-dimensional antimonene single crystals grown by van der Waals epitaxy. Nat. Commun. 7, 1-9 (2016).

34. Tsai, H.-S., Chen, C.-W., Hsiao, C.-H., Ouyang, H. \& Liang, J.-H. The advent of multilayer antimonene nanoribbons with room temperature orange light emission. Chem. Commun. 52, 8409-8412 (2016).

35. Fortin-Deschênes, M. et al. Synthesis of antimonene on germanium. Nano Lett. 17, 4970-4975 (2017)

36. $\mathrm{Wu}, \mathrm{X}$. et al. Epitaxial growth and air-stability of monolayer antimonene on PdTe2. Adv. Mater. 29, 1605407 (2017).

37. Chen, H.-A. et al. Single-crystal antimonene films prepared by molecular beam epitaxy: selective growth and contact resistance reduction of the $2 \mathrm{D}$ material heterostructure. ACS Appl. Mater. Interfaces 10, 15058-15064 (2018).

38. Sun, X. et al. van der Waals epitaxy of antimony islands, sheets, and thin films on single-crystalline graphene. ACS Nano 12, 6100-6108 (2018).

39. Liu, X. et al. High-performance asymmetric electrodes photodiode based on Sb/ WSe 2 heterostructure. Nano Res. 12, 339-344 (2019).

40. Shao, Y. et al. Epitaxial growth of flat antimonene monolayer: a new honeycomb analogue of graphene. Nano Lett. 18, 2133-2139 (2018). 
41. Mao, Y.-H. et al. Epitaxial growth of highly strained antimonene on $\mathrm{Ag}$ (111). Front. Phys. 13, 138106 (2018).

42. Ares, P. et al. Mechanical isolation of highly stable antimonene under ambient conditions. Adv. Mater. 28, 6332-6336 (2016).

43. Gibaja, C. et al. Few-layer antimonene by liquid-phase exfoliation. Angew. Chem. 128, 14557-14561 (2016).

44. Fortin-Deschênes, M. et al. Dynamics of antimonene-graphene van der Waals growth. Adv. Mater. 31, 1900569 (2019).

45. Märkl, T. et al. Engineering multiple topological phases in nanoscale Van der Waals heterostructures: realisation of a-antimonene. 2D Mater. 5, 011002 (2017).

46. Shi, Z. et al. van der Waals heteroepitaxial growth of monolayer $\mathrm{Sb}$ in a puckered honeycomb structure. Adv. Mater. 31, 1806130 (2019).

47. Akhtar, D., Vankar, V., Goel, T. \& Chopra, K. Metastable structures of liquidquenched and vapour-quenched antimony films. J. Mater. Sci. 14, 988-994 (1979).

48. Isshiki, T., Nishio, K., Saijo, H. \& Shiojiri, M. Growth and crystallographic, surface and defect structures of antimony particles deposited in a high-resolution transmission electron microscope. Thin Solid Films 237, 155-159 (1994).

49. Bernhardt, T. M., Stegemann, B., Kaiser, B. \& Rademann, K. Crystalline structures of Sb4 molecules in antimony thin films. Angew. Chem. Int. Ed. 42, 199-202 (2003).

50. Kushvaha, S., Yan, Z., Xiao, W. \& Wang, X. Surface morphology of crystalline antimony islands on graphite at room temperature. J. Phys. Condens. Matter 18, 3425 (2006)

51. Yan, Z., Kushvaha, S., Xiao, W. \& Wang, X.-S. Different-dimensional structures of antimony formed selectively on graphite. Appl. Phys. A 88, 299-307 (2007).

52. Kolobyanina, T., Kabalkina, S., Vereshchagin, L. \& Fedina, L. Investigation of the crystal structure of antimony at high pressures. Sov. Phys. JETP 28, 88-90 (1969).

53. Chang, K.-J. \& Cohen, M. L. Rhombohedral phase stability of the group-VA elements. Phys. Rev. B 33, 7371 (1986).

54. Wang, X., Kunc, K., Loa, I., Schwarz, U. \& Syassen, K. Effect of pressure on the Raman modes of antimony. Phys. Rev. B 74, 134305 (2006).

55. Zouhar, M. \& Šob, M. Ab initio study of deformed As, Sb, and Bi with an application to thin films. Phys. Rev. B 94, 184110 (2016).

56. Sun, L. et al. Band structure and thermoelectric performances of antimony under trigonal transformation. J. Appl. Phys. 125, 145102 (2019).

57. Wang, Y. \& Ding, Y. The electronic structures of group-V-group-IV hetero-bilayer structures: a first-principles study. Phys. Chem. Chem. Phys. 17, 27769-27776 (2015).

58. Li, W., Wang, X. \& Dai, X. Tunable Schottky contacts in the antimonene/graphene van der Waals heterostructures. Solid State Commun. 254, 37-41 (2017).

59. Pantelic, R. S., Meyer, J. C., Kaiser, U. \& Stahlberg, H. The application of graphene as a sample support in transmission electron microscopy. Solid State Commun. 152, 1375-1382 (2012).

60. Krivanek, O. L. et al. Atom-by-atom structural and chemical analysis by annular dark-field electron microscopy. Nature 464, 571-574 (2010).

61. Li, X. et al. Large-area synthesis of high-quality and uniform graphene films on copper foils. Science 324, 1312-1314 (2009).

62. Kidambi, P. R. et al. Observing graphene grow: catalyst-graphene interactions during scalable graphene growth on polycrystalline copper. Nano Lett. 13, 4769-4778 (2013).

63. Regan, W. et al. A direct transfer of layer-area graphene. Appl. Phys. Lett. 96, 113102 (2010).

64. $\mathrm{Wu}, \mathrm{Q}$. et al. Two-dimensional semiconducting and single-crystalline antimony trioxide directly-grown on monolayer graphene. Chem. Commun. 55, 2473-2476 (2019).

65. Deng, Y., Handoko, A. D., Du, Y., Xi, S. \& Yeo, B. S. In situ Raman spectroscopy of copper and copper oxide surfaces during electrochemical oxygen evolution reaction: identification of Culll oxides as catalytically active species. ACS Catal. 6 , 2473-2481 (2016).

66. Kidambi, P. R. et al. The parameter space of graphene chemical vapor deposition on polycrystalline Cu. J. Phys. Chem. C 116, 22492-22501 (2012).

67. Bayer, B. C. et al. Atomic-scale in situ observations of crystallization and restructuring processes in two-dimensional MoS2 films. ACS Nano 12, 8758-8769 (2018).

68. Elibol, K. et al. Process pathway controlled evolution of phase and Van-der-Waals epitaxy in In/ln2O3 on graphene heterostructures. Adv. Funct. Mater. 30, 2003300 (2020).

69. Elibol, K. et al. Single indium atoms and few-atom indium clusters anchored onto graphene via silicon heteroatoms. https://arxiv.org/abs/2009.00063 (2020).

70. Rathore, J. \& Mahapatra, S. Formation of antimonene nanoribbons by molecular beam epitaxy. 2D Mater. 7, 045003 (2020).

71. Hummel, S. et al. Direct visualization of local deformations in suspended fewlayer graphene membranes by coupled in situ atomic force and scanning electron microscopy. Appl. Phys. Lett. 118, 103104 (2021).

72. Liu, Y., Huang, Y. \& Duan, X. Van der Waals integration before and beyond twodimensional materials. Nature 567, 323-333 (2019).
73. Kim, Y. et al. Remote epitaxy through graphene enables two-dimensional material-based layer transfer. Nature 544, 340-343 (2017).

74. Niu, T. et al. Modulating epitaxial atomic structure of antimonene through interface design. Adv. Mater. 31, 1902606 (2019).

75. Niu, T. et al. Large-scale synthesis of strain-tunable semiconducting antimonene on copper oxide. Adv. Mater. 32, 1906873 (2020).

76. Ohring, M. Materials Science of Thin Films (Elsevier, 2001).

77. Zhang, S. et al. Antimonene oxides: emerging tunable direct bandgap semiconductor and novel topological insulator. Nano Lett. 17, 3434-3440 (2017).

78. Wolff, S., Gillen, R., Assebban, M., Abellán, G. \& Maultzsch, J. Two-dimensional antimony oxide. Phys. Rev. Lett. 124, 126101 (2020).

79. Wu, Q. \& Song, Y. J. The environmental stability of large-size and single-crystalline antimony flakes grown by chemical vapor deposition on SiO 2 substrates. Chem. Commun. 54, 9671-9674 (2018).

80. Fuchs, G. et al. Electron irradiation effects in amorphous antimony thin films obtained by cluster-beam deposition. Philos. Mag. B 63, 715-725 (1991).

81. Assebban, M. et al. Unveiling the oxidation behavior of liquid-phase exfoliated antimony nanosheets. 2D Mater. 7, 025039 (2020).

82. Bat-Erdene, M. et al. Surface oxidized two-dimensional antimonene nanosheets for electrochemical ammonia synthesis under ambient conditions. J. Mater. Chem. A 8, 4735-4739 (2020).

83. Sun, K., Liu, J. \& Browning, N. Correlated atomic resolution microscopy and spectroscopy studies of Sn (Sb) O2 nanophase catalysts. J. Catal. 205, 266-277 (2002).

84. Bouchet, D., Roy, E., Yu-Zhang, K. \& Leprince-Wang, Y. TEM and EELS studies of electrodeposited antimony nanowires. Eur. Phys. J. Appl. Phys. 30, 193-199 (2005).

85. Wang, Y. W. et al. Antimony nanowires self-assembled from Sb nanoparticles. J. Phys. Chem. B 108, 16723-16726 (2004).

86. Gass, M. H. et al. Free-standing graphene at atomic resolution. Nat. Nanotechnol. 3, 676-681 (2008).

87. Rafiee, J. et al. Wetting transparency of graphene. Nat. Mater. 11, 217-222 (2012).

88. Bayer, B. C., Aria, A. I., Eder, D., Hofmann, S. \& Meyer, J. C. Resolving the nucleation stage in atomic layer deposition of hafnium oxide on graphene. https://arxiv.org/ abs/1909.00712 (2019).

89. Predel, B. Sb-W (antimony-tungsten). in Pu-Re-Zn-Zr, 'Phase Equilibria, Crystallographic and Thermodynamic Data of Binary Alloys' of Landolt-Börnstein Group IV Physical Chemistry (Springer, 1998).

90. Abràmoff, M. D., Magalhães, P. J. \& Ram, S. J. Image processing with ImageJ. Biophotonics Int. 11, 36-42 (2004).

91. Elibol, K. et al. Visualising the strain distribution in suspended two-dimensional materials under local deformation. Sci. Rep. 6, 28485 (2016).

92. Nečas, D. \& Klapetek, P. Gwyddion: an open-source software for SPM data analysis. Cent. Eur. J. Phys. 10, 181-188 (2012).

93. Momma, K. \& Izumi, F. VESTA 3 for three-dimensional visualization of crystal, volumetric and morphology data. J. Appl. Crystallogr. 44, 1272-1276 (2011).

94. Mozharivskyj, Y., Pecharsky, A. O., Bud'ko, S. \& Miller, G. J. A promising thermoelectric material: Zn4Sb3 or Zn6- $\delta \mathrm{Sb} 5$. Its composition, structure, stability, and polymorphs. Structure and stability of Zn1- SSb. Chem. Mater. 16, 1580-1589 (2004).

95. Svensson, C. Refinement of the crystal structure of cubic antimony trioxide, Sb2O3. Acta Crystallogr. B 31, 2016-2018 (1975).

96. Singh, D., Gupta, S. K., Sonvane, Y. \& Lukačević, I. Antimonene: a monolayer material for ultraviolet optical nanodevices. J. Mater. Chem. C 4, 6386-6390 (2016).

\section{ACKNOWLEDGEMENTS}

B.C.B, K.E., S.H., and C.M. acknowledge support from the Austrian Research Promotion Agency (FFG) under project 860382 -VISION. The authors acknowledge use of the facilities at the University Service Centre for Transmission Electron Microscopy (USTEM), Vienna University of Technology (TU Wien), Austria, for parts of this work. The authors acknowledge the TU Wien Bibliothek for financial support through its Open Access Funding Program. The authors also thank TU Wien's Doctoral College TU-D for partial support.

\section{AUTHOR CONTRIBUTIONS}

T.G., D.E., and B.C.B. planned the experiments. T.G. and K.E. carried out synthesis. T.G. performed all (S)TEM, SEM, EDX, VEELS, and Raman characterization with input from M.S.-P., C.M., and J.C.M. Author G.H. carried out FIB preparations. S.H. performed AFSEM measurements. T.G. analyzed all data with input from B.C.B. and K.E. Authors T.G. and B.C.B. wrote the manuscript with inputs from all authors. 


\section{COMPETING INTERESTS}

At the time of performing of this study, S.H. had been affiliated with GETec Microscopy $\mathrm{GmbH}$, Austria.

\section{ADDITIONAL INFORMATION}

Supplementary information The online version contains supplementary material available at https://doi.org/10.1038/s41699-021-00230-3.

Correspondence and requests for materials should be addressed to D.E. or B.C.B.

Reprints and permission information is available at http://www.nature.com/ reprints

Publisher's note Springer Nature remains neutral with regard to jurisdictional claims in published maps and institutional affiliations.
Open Access This article is licensed under a Creative Commons Attribution 4.0 International License, which permits use, sharing, adaptation, distribution and reproduction in any medium or format, as long as you give appropriate credit to the original author(s) and the source, provide a link to the Creative Commons license, and indicate if changes were made. The images or other third party material in this article are included in the article's Creative Commons license, unless indicated otherwise in a credit line to the material. If material is not included in the article's Creative Commons license and your intended use is not permitted by statutory regulation or exceeds the permitted use, you will need to obtain permission directly from the copyright holder. To view a copy of this license, visit http://creativecommons. org/licenses/by/4.0/.

(c) The Author(s) 2021 